\title{
Space-Time Properties as Quantum Effects. Restrictions Imposed by Grothendieck's Scheme Theory
}

\author{
Leonid Lutsev \\ Ioffe Physical-Technical Institute, Russian Academy of Sciences, St. Petersburg, Russia \\ Email: 1_lutsev@mail.ru
}

How to cite this paper: Lutsev, L. (2019) Space-Time Properties as Quantum Effects. Restrictions Imposed by Grothendieck's Scheme Theory. Journal of Modern Physics, 10, 795-823.

https://doi.org/10.4236/jmp.2019.107054

Received: May 14, 2019

Accepted: June 21, 2019

Published: June 24, 2019

Copyright ( 2019 by author(s) and Scientific Research Publishing Inc. This work is licensed under the Creative Commons Attribution International License (CC BY 4.0).

http://creativecommons.org/licenses/by/4.0/

\section{cC) (i) Open Access}

\begin{abstract}
In this paper we consider properties of the four-dimensional space-time manifold $\mathcal{M}$ caused by the proposition that, according to the scheme theory, the manifold $\mathcal{M}$ is locally isomorphic to the spectrum of the algebra $\mathcal{A}$, $\mathcal{M} \cong \operatorname{Spec}(\mathcal{A})$, where $\mathcal{A}$ is the commutative algebra of distributions of quantum-field densities. Points of the manifold $\mathcal{M}$ are defined as maximal ideals of density distributions. In order to determine the algebra $\mathcal{A}$, it is necessary to define multiplication on densities and to eliminate those densities, which cannot be multiplied. This leads to essential restrictions imposed on densities and on space-time properties. It is found that the only possible case, when the commutative algebra $\mathcal{A}$ exists, is the case, when the quantum fields are in the space-time manifold $\mathcal{M}$ with the structure group $S O(3,1)$ (Lorentz group). The algebra $\mathcal{A}$ consists of distributions of densities with singularities in the closed future light cone subset. On account of the local isomorphism $\mathcal{M} \cong \operatorname{Spec}(\mathcal{A})$, the quantum fields exist only in the space-time manifold with the one-dimensional arrow of time. In the fermion sector the restrictions caused by the possibility to define the multiplication on the densities of spinor fields can explain the chirality violation. It is found that for bosons in the Higgs sector the charge conjugation symmetry violation on the densities of states can be observed. This symmetry violation can explain the matter-antimatter imbalance. It is found that in theoretical models with non-abelian gauge fields instanton distributions are impossible and tunneling effects between different topological vacua $|n\rangle$ do not occur. Diagram expansion with respect to the $\mathcal{A}$-algebra variables is considered.
\end{abstract}

\section{Keywords}

Space-Time Properties, Quantum Field, Arrow of Time, Chirality, Algebra of 
Distributions, Symmetry Violation

\section{Introduction}

The origin of the arrow of time, the possibility of physics in multiple time dimensions, the violation of the parity principle, and the matter-antimatter imbalance are ones of the most exciting and difficult challenges of physics.

Physics in multiple time dimensions leads to new insights and, at the same time, contains theoretical problems. According to the assertion written in [1] [2] [3], extra time dimensions give new hidden symmetries that conventional one time physics does not capture, implying the existence of a more unified formulation of physics that naturally supplies the hidden information. At the same time, it notes that all but the $(3+1)$-dimensional one might correspond to "dead worlds", devoid of observers, and we should find ourselves inhabiting a $(3+1)$ -dimensional space-time [4]. The natural description of the $(3+1)$-space-time with the one-dimensional time can be provided on the base of the Clifford geometric algebra [5]. In the opposite case of multidimensional time, the violation of the causal structure of the space-time and the movement backwards in the time dimensions are possible [6]. A particle can move in the causal region faster than the speed of light in vacuum. This leads to contradictoriness of the multidimensional time theory and, at present, these problems have not been solved.

The arrow of time is the one-way property of time which has no analogue in space. The asymmetry of time is explained by large numbers of theoretical models-by the Second law of thermodynamics (the thermodynamic arrow of time), by the direction of the universe expansion (the cosmological arrow), by the quantum uncertainty and entanglement of quantum states (the quantum source of time), and by the perception of a continuous movement from the known (past) to the unknown (future) (the psychological time arrow) [7]-[13]. At present, there is not a satisfactory explanation of the arrow of time and this problem is far from being solved.

The discrete symmetry of the space reflection $P$ of the space-time and the charge conjugation $C$ may be used to characterize the properties of chiral systems. The violation of the space reflection $P$ exhibits as the chiral symmetry breaking-only left-handed particles and right-handed anti-particles could be observed [14] [15] [16]. The cause of the parity violation is not clear.

The matter-antimatter imbalance remains as one of the unsolved problems. The amount of $C P$ violation in the Standard Model is insufficient to account for the observed baryon asymmetry of the universe. At present, the hope to explain the matter-antimatter imbalance is set on the $C P$ violation in the Higgs sector [17] [18] [19] [20] [21].

In this paper we consider the above-mentioned space-time properties (the arrow of time, multiple time dimensions, and the chirality violation), the violation of the charge conjugation, and find that in the framework of the scheme theory 
these properties can be determined by the spectrum of the commutative algebra $\mathcal{A}$ of distributions of quantum-field densities. Points of the space-time manifold $\mathcal{M}$ are defined as maximal ideals of quantum-field density distributions. The scheme theory imposes restrictions on space-time properties. Schemes were introduced by Alexander Grothendieck with the aim of developing the formalism needed to solve deep problems of algebraic geometry [22]. This led to the evolution of the concept of space [23]. The space is associated with a spectrum of a commutative algebra or, in other words, with a set of all prime ideals. In the case of the classical physics, the commutative algebra is the commutative ring of functions. In contrast with the classical physics, quantum fields are determined by equations on functionals [24] [25] [26] [27]. Quantum-field densities are linear functionals of auxiliary fields and, consequently, are distributions. There are many restrictions to construct the commutative algebra of distributions. In the common case, multiplication on distributions cannot be defined and depends on their wavefront sets. In the microlocal analysis the wavefront set $\mathrm{WF}(u)$ characterizes the singularities of a distribution $u$, not only in space, but also with respect to its Fourier transform at each point. The term wavefront was coined by Lars Hörmander [28]. It should be noted that the microlocal analysis has resulted in the recent progress in the renormalized quantum field theory in curved space-time [29] [30] [31] [32]. In our case, the possibility to define multiplication on distributions leads to essential restrictions imposed on densities forming the algebra $\mathcal{A}$. The spectrum of the algebra $\mathcal{A}$ is locally isomorphic to the space-time manifold $\mathcal{M}, \mathcal{M} \cong \operatorname{Spec}(\mathcal{A})$, and characterizes its properties such as the one-dimensional arrow of time, the chirality violation and the structure group of the space-time manifold $\mathcal{M}$. One can say that the space-time is determined by matter.

The principal assertion of the paper is the proposition that, according to the scheme theory, the manifold $\mathcal{M}$ is locally isomorphic to the spectrum of the algebra $\mathcal{A}, \mathcal{M} \cong \operatorname{Spec}(\mathcal{A})$, where $\mathcal{A}$ is the commutative algebra of distributions of quantum-field densities. So, the paper is organized as follows. In order to find distributions of quantum-field densities, in Section II we derive differential equations for the densities of quantum fields from the Schwinger equation and find that wavefronts of the quantum-field density distributions are determined by characteristics of the matrix differential operator and by wavefronts of distributions of composite fields (higher order functional derivatives defined at a point). The quantum fields contain fermion, boson (Higgs), and gauge field components. Multiplication on the quantum-field densities and the commutative algebra $\mathcal{A}$ of distributions of fermion and boson densities are considered in Section III. It is found that the only possible case, when the commutative algebra $\mathcal{A}$ of distributions of quantum-field densities exists, is the case, when the quantum fields are in the space-time manifold $\mathcal{M}$ with the structure group $S O(3,1)$ (Lorentz group) and the time is one-dimensional. The asymmetry of time, the chirality violation of spinor fields, and the charge conjugation symmetry violation in the boson sector are the necessary conditions for the existence of 
the algebra $\mathcal{A}$. The quantum fields exist only in the space-time manifold with the one-dimensional arrow of time and with chirality and charge conjugation symmetry violations. In Section IV we consider possibility to define a multiplication operation on instanton density distributions in theoretical models with non-abelian gauge fields. It is found that instanton distributions are impossible and, therefore, tunneling effects between different topological vacua $|n\rangle$ do not occur. This leads to the zero value of the Pontryagin index $Q$ and to the zero neutron electric dipole moment. Fermion, boson (Higgs) and gauge field density distributions satisfying the restriction requirements considered in Sections III and IV are generators of the algebra $\mathcal{A}$. Ideals, localization, the spectrum of the density distribution algebra $\mathcal{A}$ and the scheme $\left(\mathcal{M}, \mathcal{A}_{\mathcal{M}}\right)$ are considered in Section V. If the algebra $\mathcal{A}$ can be determined and is the component of the scheme, then the space-time manifold $\mathcal{M}$ with quantum fields exists. Otherwise, the space-time manifold is devoid of matter and, consequently, does not exist. In Section VI we consider diagram expansion with respect to auxiliary fields and find that wavefronts of distributions of composite fields at a point introduced in Section II are included in the characteristics of the matrix differential operator and wavefront sets of quantum-field density distributions are located on the light cone. Diagram expansion with respect to the $\mathcal{A}$-algebra variables are considered in Section VII.

\section{Quantum-Field Equations}

Quantum fields are determined by equations on functionals. In this section we consider singularities of the linear components $w^{\zeta}(x)$ (densities) of the functional solution

$$
\begin{aligned}
& Z(q)=\sum_{\zeta} \int w^{\zeta}(x) q^{\zeta}(x) \mathrm{d} x \\
& +\sum_{n>1} \sum_{\zeta_{1} \cdots \zeta_{n}} \int \cdots \int G^{\zeta_{1} \cdots \zeta_{n}}\left(x_{1} \cdots x_{n}\right) q^{\zeta_{1}}\left(x_{1}\right) \cdots q^{\zeta_{n}}\left(x_{n}\right) \mathrm{d} x_{1} \cdots \mathrm{d} x_{n},
\end{aligned}
$$

where $q^{\zeta}(x)$ is the auxiliary fields, $G^{\zeta_{1} \cdots \zeta_{n}}\left(x_{1} \cdots x_{n}\right)$ are the higher order distributions $(n>1)$. For this purpose, we derive differential equations for the densities of quantum fields from the Schwinger equation. Let us consider fields $\Psi$ on the 4-dimensional space-time manifold $\mathcal{M}$

$$
\Psi(x)=\left\{\Psi^{\zeta}(x)\right\}=\left\{\psi^{\alpha}(x), \bar{\psi}^{\alpha}(x), \varphi^{n}(x), \varphi^{+n}(x), A_{\mu}^{a}(x)\right\},
$$

where $\psi^{\alpha}(x), \bar{\psi}^{\alpha}(x)$ are the fermion (spinor) fields, $\varphi^{n}(x), \varphi^{+n}(x)$ are the bosons (for example, Higgs bosons), and $A_{\mu}^{a}(x)$ are the gauge field potentials. In relation (2) and in the all following relations Greek letter indices $\alpha$ and $\beta$ enumerate types of fermions, $\mu, v$ and $\rho$ are indices of the space-time variables, Latin letters $n, m, l$ enumerate types of bosons, and $a, b, c$ are the gauge indices, respectively. $\zeta=\{\alpha, n, a\}$ is the multiindex.

Since wavefronts of distributions can be localized [33] and a differential manifold locally resembles Euclidian space near each point, we consider the case when the space-time manifold $\mathcal{M}$ is the 4-dimensional Euclidian (pseu- 
do-Euclidian) space with the Euclidian metric tensor $g^{\mu \nu}$. In order to derive quantum-field equations, we consider the action of the fields $\Psi$ on the manifold $\mathcal{M}$ [14] [15] [34]

$$
S(\Psi)=\int_{\mathcal{M}} L(\Psi(x)) \mathrm{d} x
$$

with the Lagrangian

$$
\begin{aligned}
L(\Psi(x))= & -\frac{1}{4} F_{\mu \nu}^{a}(x) F^{\mu v a}(x)+i \bar{\psi}^{\alpha}(x) \gamma^{\mu} \nabla_{\mu \beta}^{\alpha} \psi^{\beta}(x) \\
& -m^{(\alpha)} c \bar{\psi}^{\alpha}(x) \psi^{\alpha}(x)-\mu_{\alpha \beta n} \bar{\psi}^{\alpha}(x) \varphi^{n}(x) \psi^{\beta}(x) \\
& -\mu_{\alpha \beta n}^{*} \bar{\psi}^{\alpha}(x) \varphi^{+n}(x) \psi^{\beta}(x)+\bar{\nabla}_{\mu n}^{l} \varphi^{+n}(x) \nabla_{m}^{\mu l} \varphi^{m}(x) \\
& -m^{(n) 2} c^{2} \varphi^{+n}(x) \varphi^{n}(x)+v\left(\varphi^{+n}(x) \varphi^{n}(x)\right)^{2},
\end{aligned}
$$

where

$$
\begin{gathered}
F_{\mu \nu}^{a}=\partial_{\mu} A_{\nu}^{a}-\partial_{\nu} A_{\mu}^{a}+e_{j(a)} C_{b c}^{a} A_{\mu}^{b} A_{\nu}^{c}, \\
F^{a \mu \nu}=g^{\mu \rho} g^{v \sigma} F_{\rho \sigma}^{a},
\end{gathered}
$$

are the intensity of the gauge fields,

$$
\gamma^{\mu} \gamma^{v}+\gamma^{v} \gamma^{\mu}=2 g^{\mu v}
$$

are Dirac matrices. $\mu_{\alpha \beta n}$ and $\mu_{\alpha \beta n}^{*}$ are the Yukawa interaction constants. $v$ is the boson interaction constant. $m^{(\alpha)}$ and $m^{(n)}$ are masses of fermions and bosons, respectively. $c$ is the light velocity.

$$
\begin{gathered}
\nabla_{\mu \beta}^{\alpha}=\hbar \partial_{\mu} \delta_{\beta}^{\alpha}-i e_{j(a)} T_{a \beta}^{\alpha} A_{\mu}^{a}, \\
\nabla_{\mu m}^{l}=\hbar \partial_{\mu} \delta_{m}^{l}-i e_{j(a)} \tau_{a m}^{l} A_{\mu}^{a}, \quad \nabla_{m}^{\mu l}=g^{\mu \nu} \nabla_{v m}^{l}, \\
\bar{\nabla}_{\mu m}^{l}=\hbar \partial_{\mu} \delta_{m}^{l}+i e_{j(a)} \tau_{a m}^{* l} A_{\mu}^{a},
\end{gathered}
$$

$T_{a}=\left\|T_{a \beta}^{\alpha}\right\|$ is the gauge matrix with the commutation relation $\left[T_{a}, T_{b}\right]=i C_{a b}^{c} T_{c}$ acting on spinors as $\psi^{\prime}=\exp \left(i \sigma^{a} T_{a}\right) \psi, \sigma^{a}$ is an arbitrary real number. $\tau_{a}=\left\|\tau_{a n}^{m}\right\|$ is the gauge matrix with the commutation relation $\left[\tau_{a}, \tau_{b}\right]=i C_{a b}^{c} \tau_{c}$ acting on bosons as $\varphi^{\prime}=\exp \left(i \sigma^{a} \tau_{a}\right) \varphi$. It is supposed that the summation in relation (3) and in the all following relations is performed over all repeating indices. $e_{j(a)}$ is the charge corresponding to the $j$ factor of the direct decomposition of the gauge group $\mathcal{G}=\prod_{j} \mathcal{G}_{j}(=S U(3) \times S U(2) \times U(1))$. If the index $a$ of operators $T_{a}$ and $\tau_{a}$ belongs to the subgroup $\mathcal{G}_{j}$, then in the charge $e_{j(a)}$ $j(a)=j$.

For derivation of the quantum-field Schwinger equation it needs to add the linear term $(q, \Psi)$ with auxiliary fields

$$
\begin{aligned}
& q(x)=\left\{q^{\zeta}(x)\right\}=\left\{q_{(\Psi)}^{\alpha}(x), q_{(\bar{\psi})}^{\alpha}(x), q_{(\varphi)}^{n}(x), q_{\left(\varphi^{+}\right)}^{n}(x), q_{(A) \mu}^{a}(x)\right\} \text { to the action } \\
& S(\Psi) \\
& \bar{S}(\Psi, q)=S(\Psi)+(q, \Psi)
\end{aligned}
$$

where 


$$
\begin{aligned}
(q, \Psi)= & \int_{\mathcal{M}}\left(q_{(\psi)}^{\alpha}(x) \psi^{\alpha}(x)+q_{(\bar{\psi})}^{\alpha}(x) \bar{\psi}^{\alpha}(x)+q_{(\varphi)}^{n}(x) \varphi^{n}(x)\right. \\
& \left.+q_{\left(\varphi^{+}\right)}^{n}(x) \varphi^{+n}(x)+q_{(A) \mu}^{a}(x) A_{\mu}^{a}(x)\right) \mathrm{d} x .
\end{aligned}
$$

For fields $\varphi^{n}(x), \varphi^{+n}(x)$, and $A_{\mu}^{a}(x)$ the auxiliary fields $q^{\zeta}(x)$ are simple variables and for fields $\psi^{\alpha}(x)$ and $\bar{\psi}^{\alpha}(x)$ the auxiliary fields are Grassmanian ones, respectively. Then, the Schwinger equation is written in the form [24] [25]

$$
\left\{\left.\frac{\vec{\delta} S(\Psi)}{\delta \Psi^{\zeta}(x)}\right|_{\Psi^{\zeta}(x)=\hbar \vec{\delta} / \delta i q^{\zeta}(x)}+q^{\zeta}(x)\right\} Z(q)=0,
$$

where $\vec{\delta} / \delta \Psi$ is the functional derivative on the left, $Z(q)$ is the generating functional. The derivatives $\vec{\delta} S(\Psi) / \delta \Psi^{\zeta}(x)$ have been written as

$$
\begin{aligned}
& \frac{\vec{\delta} S}{\delta \bar{\psi}^{\alpha}(x)}= i \gamma^{\mu} \nabla_{\mu \beta}^{\alpha} \psi^{\beta}(x)-m^{(\alpha)} c \psi^{\alpha}(x)-\mu_{\alpha \beta n} \varphi^{n}(x) \psi^{\beta}(x) \\
&-\mu_{\alpha \beta n}^{*} \varphi^{+n}(x) \psi^{\beta}(x), \\
& \frac{\vec{\delta} S}{\delta \psi^{\alpha}(x)}= i \bar{\psi}^{\beta}(x) \gamma^{\mu}\left(\hbar \bar{\partial}_{\mu} \delta_{\alpha}^{\beta}+i e_{j(a)} T_{a \alpha}^{\beta} A_{\mu}^{a}(x)\right)+m^{(\alpha)} c \bar{\psi}^{\alpha}(x) \\
&+\mu_{\beta \alpha n} \varphi^{n}(x) \bar{\psi}^{\beta}(x)+\mu_{\beta \alpha n}^{*} \varphi^{+n}(x) \bar{\psi}^{\beta}(x), \\
& \frac{\delta S}{\delta \varphi^{m}(x)}=-\bar{\nabla}_{\mu k}^{m} \bar{\nabla}_{n}^{\mu k} \varphi^{+n}(x)-m^{(m) 2} c^{2} \varphi^{+m}(x) \\
&+2 v \varphi^{m}(x) \varphi^{+m 2}(x)-\mu_{\alpha \beta m} \bar{\psi}^{\alpha}(x) \psi^{\beta}(x), \\
& \frac{\delta S}{\delta \varphi^{+n}(x)}-\nabla_{\mu k}^{n} \nabla_{m}^{\mu k} \varphi^{m}(x)-m^{(n) 2} c^{2} \varphi^{n}(x) \\
&+2 v \varphi^{n 2}(x) \varphi^{+n}(x)-\mu_{\alpha \beta n}^{*} \bar{\psi}^{\alpha}(x) \psi^{\beta}(x), \\
& \frac{\delta S \quad}{\delta A_{\mu}^{a}(x)}\left(\partial_{\nu} \delta_{b}^{a}+e_{j(a)} C_{c b}^{a} A_{\nu}^{c}(x)\right) F^{b v \mu}(x)+\bar{\psi}^{\alpha}(x) \gamma^{\mu} T_{a \beta}^{\alpha} \psi^{\beta}(x) \\
&-i\left(-\tau_{a n}^{* k} \varphi^{+n}(x) \nabla_{m}^{\mu k} \varphi^{m}(x)+\bar{\nabla}_{n}^{\mu k} \varphi^{+n}(x) \tau_{a m}^{k} \varphi^{m}(x)\right) .
\end{aligned}
$$

In order to get the Schwinger equation we should substitute of derivatives $\hbar \vec{\delta} / \delta i q^{\zeta}(x)$ for $\Psi^{\zeta}(x)$ in relations (5). The formal solution of the Schwinger Equation (4) is the functional integral

$$
Z(q)=\int \exp \left[\frac{i}{\hbar}(S(\Psi)+(q, \Psi))\right] D \Psi .
$$

We consider densities of the quantum fields $\psi^{\alpha}(x), \bar{\psi}^{\alpha}(x), \varphi^{n}(x)$, $\varphi^{+n}(x)$, and $A_{\mu}^{a}(x)$ in the expansion (1)

$$
w^{\zeta}(x)=\left.\frac{\vec{\delta} Z(q)}{\delta q^{\zeta}(x)}\right|_{q \rightarrow 0} .
$$

Taking into account the form of the functional $Z(q)$ (1) and the form of the Lagrangian (3), from the Schwinger Equation (4) we can obtain differential equ- 
ations for the densities

$$
\left.w(x)=\left\{w^{\zeta}(x)\right\}=\left\{w_{(\psi)}^{\alpha}(x), w_{(\bar{\psi})}^{\alpha}(x)\right), w_{(\varphi)}^{n}(x), w_{\left(\varphi^{+}\right)}^{n}(x), w_{(A) \mu}^{a}(x)\right\},
$$

which can be written in the form

$$
\begin{gathered}
w_{(\bar{\psi})}^{\alpha}(x)\left(i \hbar \gamma^{\mu} \bar{\partial}_{\mu}+m^{(\alpha)} c\right)=B_{(\bar{\psi})}^{\alpha}\left(w^{\zeta_{(n)}(\mathrm{comp})}(x)\right) \\
\left(i \hbar \gamma^{\mu} \vec{\partial}_{\mu}-m^{(\alpha)} c\right) w_{(\psi)}^{\alpha}(x)=B_{(\psi)}^{\alpha}\left(w^{\zeta_{(n)}(\mathrm{comp})}(x)\right) \\
\left(\hbar^{2} \square+m^{(n) 2} c^{2}\right) w_{(\varphi)}^{n}(x)=B_{(\varphi)}^{n}\left(w^{\zeta_{(n)}(\mathrm{comp})}(x)\right) \\
\left(\hbar^{2} \square+m^{(n) 2} c^{2}\right) w_{\left(\varphi^{+}\right)}^{n}(x)=B_{\left(\varphi^{+}\right)}^{n}\left(w^{\zeta_{(n)}(\mathrm{comp})}(x)\right) \\
\square w_{(A) \mu}^{a}(x)=B_{(A) \mu}^{a}\left(w^{\zeta_{(n)}(\mathrm{comp})}(x)\right),
\end{gathered}
$$

where

$$
\square(\cdot)=g^{\mu v} \frac{\partial^{2}(\cdot)}{\partial x^{\mu} x^{v}}
$$

is the d'Alembert operator; $B^{\zeta}=\left\{B_{(\psi)}^{\alpha}, B_{(\bar{\psi})}^{\alpha}, B_{(\varphi)}^{n}, B_{\left(\varphi^{+}\right)}^{n}, B_{(A) \mu}^{a}\right\}$ are polynomials of distributions $w^{\zeta_{(n)}(\mathrm{comp})}(x)$ of composite fields $\bar{\psi}^{\alpha}(x) \psi^{\beta}(x)$, $\varphi^{n}(x) \psi^{\beta}(x), \varphi^{+n}(x) \varphi^{m}(x), A_{\mu}^{a}(x) A_{v}^{b}(x), \cdots$. Distributions $w^{\zeta_{(n)}(\text { comp })}(x)$ $\left(\zeta_{(n)}=\left\{\zeta_{1}, \cdots, \zeta_{n}\right\}, n \geq 2\right)$ are defined as higher order functional derivatives at a point

$$
w^{\zeta_{(n)}(\text { comp })}(x)=\left.\underbrace{\frac{\delta^{n} W}{\delta q^{\zeta_{1}}\left(x_{1}\right) \cdots \delta q^{\zeta_{n}}\left(x_{n}\right)}}_{n}\right|_{x_{1}, \cdots, x_{n} \rightarrow x}
$$

Equations (7) can be written in the form

$$
\mathcal{N}_{\zeta}^{\eta} w^{\zeta}(x)=B^{\eta}\left(w^{\zeta(n)(\text { comp })}(x)\right)
$$

where $\mathcal{N}_{\zeta}^{\eta}=\sum_{p=0}^{k} a_{p \zeta}^{\eta} \partial_{x}^{p}$ is the matrix differential operator. Solutions of the Schwinger equation (4) determined the generating functional $Z(q)$ and solutions of Equations (7), (8) can be found in the approximate form by the diagram technique [25] [26] [27], which will be considered in Sections VI and VII. In the common case, solutions of Equations (7) and (8) are distributions and have singularities. The question is: which distributions of the densities $w^{\zeta}(x)$ can be multiplied and, therefore, form a commutative algebra? We consider the densities $w^{\zeta}(x)$ expressed in the oscillatory--integral form [33] [35] [36]

$$
w^{\zeta}(x)=\int_{T^{*} \mathcal{M}} F^{\zeta}(x, \chi) \exp \left[i \sigma^{\zeta}(x, \chi)\right] \mathrm{d} \chi,
$$

where $T^{*} \mathcal{M}$ is the cotangent bundle over the space-time manifold $\mathcal{M}$, $\sigma^{\zeta}(x, \chi)$ is the phase function, $F^{\zeta}(x, \chi)$ is the amplitude, $\chi$ is the covector, and $(x, \chi) \in T^{*} \mathcal{M}$. It should be noted that relation (9) defines Lagrangian distributions, which form the subset of the space of all distributions $D^{\prime}(\mathcal{M})$. 
Any Lagrangian distribution can be represented locally by oscillatory integrals [36]. Conversely, any oscillatory integral is a Lagrangian distribution. We consider the case of the real linear phase function of $\chi$

$$
\sigma^{\zeta}(x, \chi)=k^{\zeta} \chi_{v} x_{v},
$$

where $k^{\zeta}$ is a coefficient. Our consideration of the case of Lagrangian distributions is motivated by the statement that, if the multiplication cannot be defined on the Lagrangian distribution subset, then this operation cannot be defined on the space $D^{\prime}(\mathcal{M})$.

The wavefront set $\operatorname{WF}(u)$ of a distribution $u$ can be defined as [28] [33] [35]

$$
\operatorname{WF}(u)=\left\{(x, \xi) \in T^{*} \mathcal{M} \mid \xi \in \Gamma_{x}(u)\right\},
$$

where the singular cone $\Gamma_{x}(u)$ is the complement of all directions $\xi$ such that the Fourier transform of $u$, localized at $x$, is sufficiently regular when restricted to a conical neighborhood of $\xi$. The wavefront of the distributions $\mathrm{WF}\left(w^{\zeta}\right)$ characterizes the singularities of solutions and is determined by the wavefront of $w^{\zeta_{(n)}(\text { comp) }}(x)$ and by the characteristics of the matrix operator $\mathcal{N}_{\zeta}^{\eta}[33][35]$

$$
\mathrm{WF}\left(w^{\zeta}\right) \subset \operatorname{Char}\left(\mathcal{N}_{\zeta}^{\eta}\right) \cup \operatorname{WF}\left(B^{\eta}\left(w^{\zeta(n)(\text { comp })}\right)\right),
$$

where the characteristics $\operatorname{Char}\left(\mathcal{N}_{\zeta}^{\eta}\right)$ is the set $\left\{(x, \xi) \in T^{*} M \backslash 0\right\}$ defined by linear algebraic equations of highest power orders in the unknown Fourier transforms $\tilde{w}^{\zeta}(\xi)$

$$
\sum_{\zeta, p=\max k} a_{p \zeta}^{\eta}(-i \xi)^{p} \tilde{w}^{\zeta}(\xi)=0,
$$

where coefficients $a_{p \zeta}^{\eta}$ are defined by the matrix differential operator $\mathcal{N}_{\zeta}^{\eta}=\sum_{p=0}^{k} a_{p \zeta}^{\eta} \partial_{x}^{p}$ in Equation (8) and are equal to coefficients of linear differential operators acted on the densities $w(x)$ in equation (7). The covector $\xi \in T^{*}(x)$ lies in the cotangent cone $\Gamma_{x}$ at the point $x$. Taking into account Equations (7), we can find that

$$
\left.\operatorname{Char}\left(\mathcal{N}_{\zeta}^{\eta}\right)\right|_{x}=g^{\mu v} \xi_{\mu} \xi_{v},
$$

consequently, singularities of solutions are located on this cone. The wavefronts $\mathrm{WF}\left(w^{5(n)^{(\text {comp })}}\right)$ of composite fields are considered in Sections III and VI. In Section VI we find that in the framework of the diagram expansion wavefronts $\mathrm{WF}\left(w^{\zeta(n)(\mathrm{comp})}\right)$ are determined by $\operatorname{Char}\left(\mathcal{N}_{\zeta}^{\eta}\right)$.

Starting from the proposition that properties of the space-time manifold $\mathcal{M}$ are defined by the quantum fields $\Psi^{\zeta}(x)$ and, consequently, by the commutative algebra $\mathcal{A}$ of distributions $w^{\zeta}(x)$, in the next section we find that this statement results in essential restrictions imposed on the space-time manifold $\mathcal{M}$.

\section{Algebra of Distributions of Quantum-Field Densities. Fermion and Boson Sectors}

In order to determine points of the space-time manifold $\mathcal{M}$ by means of the 
densities $w^{\zeta}(x)$, it is necessary to define multiplication on densities, to construct the commutative algebra $\mathcal{A}$ of distributions of densities, and to find maximal ideals of this algebra. According to Ref. [33] [35], multiplication on distributions $u, v \in D^{\prime}(M)$ with wavefronts $\operatorname{WF}(u)=\{(x, \xi)\}$ and $\mathrm{WF}(v)=\{(x, \eta)\}$ is determined, if and only if

$$
\mathrm{WF}(u) \cap \mathrm{WF}^{\prime}(v)=\varnothing,
$$

where $\operatorname{WF}^{\prime}(v)$ is the image of $\operatorname{WF}(v)$ in the transformation $(x, \eta) \mapsto(x,-\eta)$ in the cone subset $\Gamma$ of the cotangent bundle $T^{*} M$. The wavefront of the product is defined as

$$
\begin{array}{r}
\mathrm{WF}(u v) \subset\{(x, \xi+\eta) \mid(x, \xi) \in \mathrm{WF}(u) \text { or } \xi=0, \\
(x, \eta) \in \mathrm{WF}(v) \text { or } \eta=0 ; \xi+\eta \neq 0\} .
\end{array}
$$

Taking into account the linear form of the phase function (10), from relations (12) and (13) we obtain restrictions on the densities $w^{\zeta}(x)$. For this purpose, we consider wavefronts of densities for the case of the 4-dimensional space-time manifold $\mathcal{M}$, when the structure group of the cotangent bundle $T^{*} \mathcal{M}$ is the Lie group $S O(4-p, p)$ with $p=0, p=1$, and $p>1$.

The fields $\Psi$ (2) are observed relative to inertial frames of reference in the space-time manifold $\mathcal{M}$. Rectilinear motion transforms the fields $\Psi$ and, consequently, their densities $w^{\zeta}(x)$ and wavefronts. This transformation can be considered as a diffeomorphism of $\mathcal{M}$ and is described by the arcwise connected part of the $S O(4-p, p)$ group. More precisely, if $f: \Omega \rightarrow \Omega^{\prime}$ is the diffeomorphism of open subsets $\Omega, \Omega^{\prime} \subset M, f_{+}: T^{*} \Omega \rightarrow T^{*} \Omega^{\prime}$ is the diffeomorphism induced by $f$, and $f_{*}: D^{\prime}(\Omega) \rightarrow D^{\prime}\left(\Omega^{\prime}\right)$ is the isomorphism, then for the distribution $u \in D^{\prime}(\Omega)$ [33] [35]

$$
\mathrm{WF}\left(f_{*} u\right)=f_{+} \mathrm{WF}(u) .
$$

Thus, rectilinear motion results in the transformation $f_{*}$ of the field densities $w^{\zeta}(x)$ induced by the arcwise connected part of the structure group and the transformation $f_{+}$of density wavefronts (14). If, as a result of these transformations, the covector $\xi \in \mathrm{WF}\left(w^{\zeta}\right) \subset T^{*} M$ changes its orientation $\xi \mapsto-\xi$, then the multiplication (13) on the density $w_{1}^{\zeta}(x)$ with the covector $\xi$ and the density $w_{2}^{\zeta}(x)$ with the covector $-\xi$ is impossible. We consider transformations of density wavefronts induced by the arcwise connected part of the structure group (14) and by discrete symmetry transformations-the time reversal and the space reflection.

\subsection{Time Reversal}

We assume that the co-ordinate variables of the space-time manifold $\mathcal{M}$ can be divided by time $c t$ and space $r$ variables, $x=\left\{c t_{1}, \cdots, c t_{p}, r_{p+1}, \cdots\right\}$

$(p=0,1,2, \cdots)$. The structure group of the space-time manifold is $S O(4-p, p)$. We consider the time reversal $T$ on the manifold $\mathcal{M}$ and the possibility of embedding of the time reversal into the arcwise connected part of the group 
$S O(4-p, p)$.

Euclidian space-time manifold with the structure group $S O(4,0)$. Let us consider the case of the time reversal on the Euclidian space-time manifold $M$ with the structure group $S O(4,0)$ of the cotangent bundle $T^{*} \mathcal{M}$. The signature of the space-time metric is $(----)$. In this case, $p=0$ and the time variable $c t$ is identical to the space one (for example, $r_{1}$ ). The image of the inversion $\xi \mapsto-\xi$ in relation (14) on wavefronts of densities can be reached by the transformation induced by the arcwise connected part of the group $S O(4,0)$ (Figure 1(a)). Thus, we always can find densities with covectors $\xi$ and $\eta$ in relation (13) such that $\xi+\eta=0$. Consequently, multiplication on distributions in the Euclidian space-time manifold with the signature of the space-time metric $(----)$ is impossible. One can only say about a partial multiplication operation on a subset of the distribution space. The analogous consideration can be carried out for manifold $\mathcal{M}$ with the structure group $S O(0,4)$ and with the signature of the space-time metric $(++++)$.

Pseudo-Euclidian space-time manifold with the structure group $S O(3,1)$. The signature of the space-time metric is $(+---)$. In accordance with relation $g^{\mu v} \xi_{\mu} \xi_{v}=0$ defining boundaries of a singular cone, the space-time manifold is separated by three regions: the future light cone $\Gamma^{(f)}$, the past light cone $\Gamma^{(p)}$, and the space-like region $\Gamma^{(s)}$ (Figure $1(\mathrm{~b})$ ). The multiplication of distributions with singularities in the region $\Gamma^{(s)}$ is impossible because of the existence of the inversion $\xi \mapsto-\xi$ reached by the arcwise connected part of the group $S O(3,1)$. Therefore, singularities can exist only in the cone $\Gamma^{(f)}$ or in the cone $\Gamma^{(p)}$. The time reversal $T$ inverts the covector $\xi \in \mathrm{WF}\left(w^{\zeta}\right) \subset T^{*} \mathcal{M}$ from the future light cone $\Gamma^{(f)}$ to the past light cone $\Gamma^{(p)}$ (Figure 1(b)). The Lorentz transformation $S O(3,1)$ acts on future and past light cones separately. Consequently, the arcwise connected part of the group $S O(3,1)$ does not contain the time inversion. So, if we exclude field density distributions $w^{\zeta}(x)$ with singularities in the past light cone $\Gamma^{(p)}$ and consider only density distributions with singularities in the future light cone $\Gamma^{(f)}$, then for these distributions the multiplication can be defined. Correspondingly, densities of states $T \Psi^{\zeta}(x)$ are forbidden. In this case, we have the one-way direction of time and there is not the symmetry of time on density distributions. The arrow of time is pointing towards the future. According to (13), the wavefront of the product of quantum-field densities in the future light cone $\Gamma^{(f)}$ is given by

$$
\mathrm{WF}\left(w^{\zeta_{1}} \cdots w^{\zeta_{n}}\right) \subset\left\{\left(x, \sum_{i}^{n} \xi^{\zeta_{i}}\right) \mid \xi^{\zeta_{i}} \in \Gamma^{(f)}, \sum_{i}^{n} \xi^{\zeta_{i}} \neq 0\right\},
$$

where $\xi^{\zeta_{i}}=\beta^{\zeta_{i}} \chi^{(i)}$ is the covector associated with the density $w^{\zeta_{i}}, \beta^{\zeta}$ is a function of field invariants, such as chirality, charge signs, charge parity, etc. Taking into account that for the Dirac adjoint spinor density $w_{(\bar{\psi})}^{\alpha}(x)$ and for the density $w_{\left(\varphi^{+}\right)}^{n}(x)$ the exponent term in the integral in relation (9) must have opposite sign than for densities $w_{(\psi)}^{\alpha}(x)$ and $w_{(\varphi)}^{n}(x)$, respectively, and, therefore, is transformed as $i \sigma^{\zeta}(x, \chi) \rightarrow-i \sigma^{\zeta}(x, \chi)$ (for $w_{(\psi)}^{\alpha}(x) \rightarrow w_{(\bar{\psi})}^{\alpha}(x)$, $\left.w_{(\varphi)}^{n}(x) \rightarrow w_{\left(\varphi^{+}\right)}^{n}(x)\right)$, we find that in the cone $\Gamma^{(f)}$ 

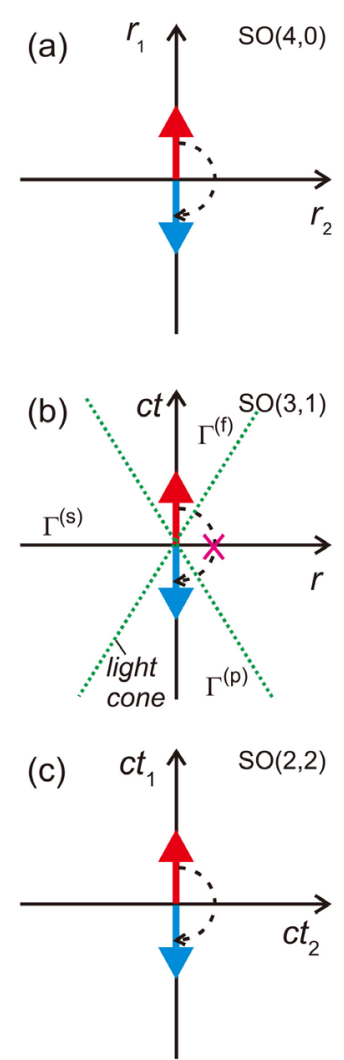

Figure 1. Transformation of the covector $\xi \mapsto-\xi$ at the time reversal of the space-time manifold $\mathcal{M}$ with the structure groups (a) $S O(4,0)$, (b) $S O(3,1)$, and (c) $S O(2,2)$. The image of the transformation $\xi \mapsto-\xi$ can be reached by means of a transformation of the arcwise connected part of groups $S O(4,0)$ and $S O(2,2)$ and is not attainable for the case of the arcwise connected part of the $S O(3,1)$ group.

$$
\begin{aligned}
& \beta_{(\psi)}>0, \quad \beta_{(\bar{\psi})}<0 \\
& \beta_{(\varphi)}>0, \quad \beta_{\left(\varphi^{+}\right)}<0 .
\end{aligned}
$$

\section{Pseudo-Euclidian space-time manifold with the structure group}

$S O(4-p, p)(p>1)$. The signatures of the space-time metric are $(++--)$ $(p=2)$ and $(+++-)(p=3)$. If the time dimension is equal to 2 or higher $(p>1)$, then the image of the time inversion of densities in the time plane $\left(c t_{1}, \cdots, c t_{p}\right)$ is attained by means of a transformation of the arcwise connected part of the group $S O(4-p, p)$ (Figure $1(\mathrm{c})$ ). In this case, one can find densities with covectors $\xi$ and $\eta$ in relation (13) such that $\xi+\eta=0$. Multiplication on distributions in this space-time manifold is impossible.

\subsection{Space Reflection and Charge Conjugation in the Fermion Sector}

Another restriction to define multiplication on the density distribution algebra is 
caused by the chirality of fermions and by the charge conjugation. Since, according to the above-mentioned subsection the pseudo-Euclidian space-time manifold with the Lorentz group $S O(3,1)$ and the arrow of time are necessary conditions for definition of multiplication on density distributions, we consider fermion distributions with singularities in the future light cone $\Gamma^{(f)}$. Right-handed and left-handed states of the Dirac fields $\psi^{\alpha}(x)$ and $\bar{\psi}^{\alpha}(x)$ are defined by projective operators $\left(1 \pm \gamma^{5}\right) / 2$ acting on a spinor [14] [15] [37]

$$
\begin{aligned}
& \psi_{R}^{\alpha}(x)=\frac{1+\gamma^{5}}{2} \psi^{\alpha}(x) \\
& \psi_{L}^{\alpha}(x)=\frac{1-\gamma^{5}}{2} \psi^{\alpha}(x) .
\end{aligned}
$$

The space reflection $P$ converts the right-handed spinor into the left-handed one, and vice versa

$$
\begin{gathered}
P \psi_{R}^{\alpha}(x)=i L_{p} \psi_{L}^{\alpha}(x) \\
P \psi_{L}^{\alpha}(x)=i L_{p} \psi_{R}^{\alpha}(x),
\end{gathered}
$$

where in the Weyl (chiral) basis

$$
L_{p}=\left(\begin{array}{ll}
0 & I \\
I & 0
\end{array}\right)
$$

and $I$ is the identity 2-matrix. $\psi_{R}^{\alpha}(x)$ and $\psi_{L}^{\alpha}(x)$ are eigenvectors of the operator $\gamma^{5}$ with the chirality $\lambda=1$ (the right-handed state) and the chirality $\lambda=-1$ (the left-handed state), respectively. Double space reflection $P^{2}$ can be regarded as $360^{\circ}$ rotation. It transforms spinors as

$$
P^{2} \psi_{\rho}^{\alpha}(x)=-\psi_{\rho}^{\alpha}(x),
$$

where $\rho=\{R, L\}$.

For fulfilment of relations (15) the coefficients $\beta_{(\psi)}$ and $\beta_{(\bar{\psi})}$ must contain a charge factor $\kappa_{c}$ such that $\beta(\psi)=1$ and $\beta(\bar{\psi})=-1$. We consider two cases, (1) $\beta=\lambda \kappa_{c}$ with the chirality $\lambda$ and (2) $\beta=\kappa_{c}$ without chirality. In order to fulfil multiplication on densities $w_{\left(\psi_{R}\right)}^{\alpha}(x), w_{\left(\psi_{L}\right)}^{\alpha}(x), w_{\left(\bar{\psi}_{R}\right)}^{\alpha}(x)$, $w_{\left(\bar{\psi}_{L}\right)}^{\alpha}(x)$ in the first case, for right-handed and left-handed states of the fermion fields we should get

$$
\begin{array}{cc}
\kappa_{c}\left(\psi_{R}^{\alpha}(x)\right)=1, & \kappa_{c}\left(\psi_{L}^{\alpha}(x)\right)=-1, \\
\kappa_{c}\left(\bar{\psi}_{R}^{\alpha}(x)\right)=-1, & \kappa_{c}\left(\bar{\psi}_{L}^{\alpha}(x)\right)=1, \\
\lambda\left(\psi_{R}^{\alpha}(x)\right)=1, & \lambda\left(\psi_{L}^{\alpha}(x)\right)=-1, \\
\lambda\left(\bar{\psi}_{R}^{\alpha}(x)\right)=1, & \lambda\left(\bar{\psi}_{L}^{\alpha}(x)\right)=-1 .
\end{array}
$$

The charge conjugation $C$ transforms $\kappa_{c}: C \kappa_{c}\left(\psi_{R}^{\alpha}(x)\right)=\kappa_{c}\left(\bar{\psi}_{R}^{\alpha}(x)\right)=-1$, $C \kappa_{c}\left(\bar{\psi}_{R}^{\alpha}(x)\right)=\kappa_{c}\left(\psi_{R}^{\alpha}(x)\right)=1, C \kappa_{c}\left(\psi_{L}^{\alpha}(x)\right)=\kappa_{c}\left(\bar{\psi}_{L}^{\alpha}(x)\right)=1$, and $C \kappa_{c}\left(\bar{\psi}_{L}^{\alpha}(x)\right)=\kappa_{c}\left(\psi_{L}^{\alpha}(x)\right)=-1$. The commutative algebra $\mathcal{A}$ of distributions contains densities $w^{\zeta}(x)$ of states $\psi_{R}^{\alpha}(x), \psi_{L}^{\alpha}(x), \bar{\psi}_{R}^{\alpha}(x), \bar{\psi}_{L}^{\alpha}(x)$, 
$C P \psi_{R}^{\alpha}(x), C P \psi_{L}^{\alpha}(x), C P \bar{\psi}_{R}^{\alpha}(x), C P \bar{\psi}_{L}^{\alpha}(x)$, their sums and products. Densities $w^{\zeta}(x)$ of states $P \psi_{R}^{\alpha}(x), P \psi_{L}^{\alpha}(x), P \bar{\psi}_{R}^{\alpha}(x), P \bar{\psi}_{L}^{\alpha}(x), C \psi_{R}^{\alpha}(x)$, $C \psi_{L}^{\alpha}(x), C \bar{\psi}_{R}^{\alpha}(x), C \bar{\psi}_{L}^{\alpha}(x)$ are forbidden and are not contained in the algebra $\mathcal{A}$. This version of the theoretical model contains particles and antiparticles and can explain the chirality violation. Quantum states of a particle and an antiparticle can be interchanged by applying the $C P$-operation.

In the second case, the fulfilment of the relation $\beta=\kappa_{c}$ with

$\kappa_{c}\left(\psi_{R}^{\alpha}(x)\right)=\kappa_{c}\left(\psi_{L}^{\alpha}(x)\right)=1$ and $\kappa_{c}\left(\bar{\psi}_{R}^{\alpha}(x)\right)=\kappa_{c}\left(\bar{\psi}_{L}^{\alpha}(x)\right)=-1$ results in forbidden densities $w^{\zeta}(x)$ of states $C \psi_{R}^{\alpha}(x), C \psi_{L}^{\alpha}(x), C \bar{\psi}_{R}^{\alpha}(x)$, and $C \bar{\psi}_{L}^{\alpha}(x)$. Wavefronts of these densities are in the past light cone $\Gamma^{(p)}$ : $\operatorname{WF}\left(w_{\left(C \psi_{R}\right)}^{\alpha}\right), \operatorname{WF}\left(w_{\left(C \psi_{L}\right)}^{\alpha}\right), \operatorname{WF}\left(w_{\left(C \bar{\psi}_{R}\right)}^{\alpha}\right)$, and $\operatorname{WF}\left(w_{\left(C \bar{\psi}_{L}\right)}^{\alpha}\right) \in \Gamma^{(p)}$. At the same time, the commutative algebra $\mathcal{A}$ of distributions contains densities $w^{\zeta}(x)$ of states $\psi_{R}^{\alpha}(x), \psi_{L}^{\alpha}(x), \bar{\psi}_{R}^{\alpha}(x), \bar{\psi}_{L}^{\alpha}(x), P \psi_{R}^{\alpha}(x), P \psi_{L}^{\alpha}(x), P \bar{\psi}_{R}^{\alpha}(x)$, and $P \bar{\psi}_{L}^{\alpha}(x)$. In this case, the theoretical model does not contain antiparticles and the chirality is not violated. In the experiment this case of the theoretical model is not observed.

\subsection{Charge Conjugation in the Boson Sector}

We consider the common case of the boson (Higgs) sector containing the quantum fields $\varphi^{n}(x)$ and $\varphi^{+n}(x)$. We assume that $\varphi^{n}(x) \neq \varphi^{+n}(x)$. By analogy with the fermion case, the covector of singularity of the density of $\varphi^{n}(x)$ is $\beta_{(\varphi)} \chi$ and the analogous covector of the density of $\varphi^{+n}(x)$ is $-\beta_{\left(\varphi^{+}\right)} \chi$, respectively. In order to fulfil relations (15) and the requirement that $\beta_{(\varphi)} \chi$, $-\beta_{\left(\varphi^{+}\right)} \chi \in \Gamma^{(f)}$, we must write the coefficient $\beta$ in the form

$\beta_{(\varphi)}=\kappa_{c}\left(\varphi^{n}(x)\right)=1$ and $\beta_{\left(\varphi^{+}\right)}=\kappa_{c}\left(\varphi^{+n}(x)\right)=-1$. The charge conjugation $C$ changes the sign of the factor $\kappa_{c}: C \kappa_{c}\left(\varphi^{n}(x)\right)=\kappa_{c}\left(\varphi^{+n}(x)\right)=-1$ and $C \kappa_{c}\left(\varphi^{+n}(x)\right)=\kappa_{c}\left(\varphi^{n}(x)\right)=1$. Thus, densities of $\varphi^{n}(x)$ and $\varphi^{+n}(x)$ can be multiplied and are included in the algebra $\mathcal{A}$. On the contrary, wavefronts of densities of states $C \varphi^{n}(x)$ and $C \varphi^{+n}(x)$ are in the past light cone $\Gamma^{(p)}$ and must be excluded. This leads to the charge conjugation symmetry violation in the boson sector.

It should be noted that in the modified theoretical models of the Higgs boson sector [17] [18] [19] [20] [21] [41] [42] [43] [44] extending the BEH model [38] [39] [40] some particles in the Higgs sector have negative charge parities and are charged. In this case, the above-mentioned $C$-violation on density distributions in the Higgs sector can explain the observed matter-antimatter imbalance.

Thus, as a result of this section, we define the multiplication on distributions of the fermion and boson (Higgs) quantum-field densities. Fermion and boson density distributions with singularities in the future light cone $\Gamma^{(f)}$ are generators of the algebra $\mathcal{A}$, which is commutative for boson densities and supercommutative for fermion ones (Grassmanian variables). The asymmetry of time ( $T$-violation), the chiral asymmetry ( $P$-violation) and the charge $(C)$ conjugation symmetry violation are caused by singularities of density distributions and these space-time manifold properties are local. In the next section we consider restric- 
tions imposed by fulfilment of the density multiplication operation for gauge fields.

\section{Gauge Fields and the Density Distribution Algebra}

Multiplication on distributions in the density distribution algebra $\mathcal{A}$ imposes restrictions on theoretical models with non-abelian gauge fields. In this section we demonstrate that instantons ensured tunneling between different topological vacua cannot be included in the algebra $\mathcal{A}$. Let us consider the gauge fields $A_{\mu}^{a}$ with the non-abelian gauge group $\mathcal{G}$ in the pseudo-Euclidian space-time manifold $\mathcal{M}$ (the Minkowski space-time) with the structure group $S O(3,1)$ and discuss the generally accepted instanton model, in which instantons are functions. At the given time $t$ the space variables form the Euclidian space $\mathbb{R}^{3}$. Adjoining the single point $\infty$, we can form the compact topological space $\mathbb{R}^{3} \cup\{\infty\}$. This one-point compactification of the 3-dimensional Euclidean space $\mathbb{R}^{3}$ is homeomorphic to the 3-sphere $\mathbb{S}^{3}$. If the local transformation $U(x, t) \in G$ of the gauge Lie group $\mathcal{G}$ is trivial at $|x| \rightarrow \infty$

$$
\lim _{|x| \rightarrow \infty} U(x, t)=1 \text {, }
$$

then the gauge potential $A_{\mu}=A_{\mu}^{a} T_{a} \quad\left(T_{a}\right.$ is the gauge matrix belonging to the Lie algebra of the group $\mathcal{G}$ ) at $|x| \rightarrow \infty$ is the pure gauge

$$
A_{\mu}=U^{-1}(x, t) \partial_{\mu} U(x, t) .
$$

and the gauge fields $F_{\mu v}^{a}$ are vanishing. The map on the gauge group $\mathcal{G}$

$$
f: \mathbb{S}^{3} \rightarrow \mathcal{G}
$$

defines the homotopy group $\pi_{3}(\mathcal{G})$. If $G=S U(N)$, then, using the Bott periodicity theorem for unitary groups $S U(N)(N \geq 2)$, one can find that [45] $[46]$

$$
\pi_{3}(S U(N))=\mathbb{Z}
$$

In this case, the gauge potential $A_{\mu}$ (16) is characterized by homotopy classes $n \in \mathbb{Z}$ of the group $\pi_{3}(S U(N))$ (17) and can be classified by the topological index [47]

$$
n=\frac{1}{24 \pi^{2}} \oint_{\mathbb{S}^{3}} \varepsilon_{\mu \nu \rho \sigma} \operatorname{tr}\left[A_{\nu} A_{\rho} A_{\sigma}\right] \mathrm{d} \sigma_{\mu},
$$

where $\varepsilon_{\mu v \rho \sigma}=(-1)^{p}, p$ is the parity of the permutation $\{\mu v \rho \sigma\}$. If the Minkowski space-time $\mathcal{M}$ can be substituted by the Euclidian space-time manifold, then the topological index (18) can be written via the Pontryagin index

$$
Q=\frac{-1}{16 \pi^{2}} \int \operatorname{tr}\left[\tilde{F}_{\mu \nu} F^{\mu \nu}\right] \mathrm{d}^{4} x,
$$

where $\tilde{F}_{\mu \nu}=\varepsilon_{\mu v \rho \sigma} F^{\rho \sigma} / 2$ is the dual fields in Euclidean space, $F^{\mu v}=F^{a \mu v} T^{a}$, and $\tilde{F}_{\mu v}=\tilde{F}_{\mu v}^{a} T^{a}$.

Taking into account (16), (17), and (18), one can find that vacuum states are characterized by the topological index $n$. The gauge operator $U_{m}$ with the to- 
pological index $m$ transforms the vacuum state [47] [48] [49]

$$
U_{m}|n\rangle=|n+m\rangle \text {. }
$$

According to [14] [15] [47] [48] [49] [50] an instanton is a gauge field configuration fulfilling the classical equations of motion in Euclidean space-time, which is interpreted as a tunneling effect between different topological vacua $|n\rangle$. Instantons are labeled by its Pontryagin index $Q(19)$. If $Q=1$, the instanton solution is written as [14] [15] [47] [48] [49] [50]

$$
A_{\mu}=\left(\frac{\rho^{2}}{\rho^{2}+\lambda^{2}}\right) U_{1}^{-1}(x, t) \partial_{\mu} U_{1}(x, t),
$$

where $\lambda$ is the scale parameter giving the instanton size, $\rho=\left.\left.\left|x^{2}+(c t)^{2}-\right| a\right|^{2}\right|^{1 / 2}, a$ is the instanton center point. One can imagine that the instanton (21) ensures tunneling between the topological vacuum $|n\rangle$ at $t \rightarrow-\infty$ and the vacuum $|n+1\rangle$ at $t \rightarrow \infty$. Taking into account that topological vacua $|n\rangle$ are connected by instanton tunneling and requiring the physical vacuum state to be stable against gauge transformations (20), one can find that the physical vacuum is

$$
|\theta\rangle=\sum_{n} \mathrm{e}^{-i n \theta}|n\rangle
$$

where $\theta$ is the phase angle. In this case, $U_{m}|\theta\rangle=\mathrm{e}^{i m \theta}|\theta\rangle$. The gauge theory with $\theta$-vacuum $(\theta \neq 0)$ (22) can be regarded as the theoretical model with the additional term proportional to $\theta Q$ in the action (3) [14] [15] [47]

$$
S \rightarrow S-i \theta Q
$$

in the case of the Euclidean space-time and

$$
S \rightarrow S+\theta Q
$$

in the case of the Minkowski space-time. $Q$ is the Pontryagin index (19). The additional term $\theta Q$ breaks $P$ and $C P$ invariance [14] [15] [47] [51].

In the instanton solution $A_{\mu}=A_{\mu}^{a} T_{a}$ (21) the gauge potentials $A_{\mu}^{a}$ are functions. Can the instanton solution (21) be generalized on the distribution space $D^{\prime}(\mathcal{M})$ ? And can instanton density distributions are multiplied? In order to answer these questions, we consider theoretical models, in which Euclidean instanton solutions have been found and the tunneling behavior has been transfered on the Minkowski space by the Wick rotation [47] [49] [50] [52] [53], and models, in which vacuum tunneling has been studied directly in the Minkowski space [54] [55].

\section{Euclidean instanton solutions.}

In order to look for a tunneling path in gauge theory which connects topologically different classical vacua $|n\rangle$, one performs an analytic continuation of the action $S(\Psi)$ (3) to imaginary (Euclidean) time $\tau=i t$ [47] [49] [50] [52] [53]. In this case, instanton solutions (21) are functions and the analytic continuation is correct. In contrast with function spaces, the analytic continuation of density distributions of quantum fields $w_{(A) \mu}^{a}(x) \in A \in D^{\prime}(\mathcal{M})$ to imaginary time 
$\tau=$ it changes their singularities. The changes in singularities are due to changes in the functional integral (6)

$$
\begin{aligned}
& Z(q)=\int \exp \left[\frac{i}{\hbar}(S(\Psi)+(q, \Psi))\right] D \Psi \\
& \rightarrow \int \exp \left[\frac{-1}{\hbar}(S(\Psi)+(q, \Psi))\right] D \Psi .
\end{aligned}
$$

Let us consider these changes in distributions $w_{(A) \mu}^{a}(x)$ in detail. Suppose that a gauge field has the topological vacuum $|n\rangle$ at $t \rightarrow-\infty$. In order to find a tunneling path, we consider $t>-\infty$ and perform the complexification $t^{c} \rightarrow t+i \tau \quad(t, \tau \in \mathfrak{R})$. In this case, the time is 2 -dimensional. According to Figure 1 (c) (Section III), one can find densities $w_{(A) \mu}^{a}(x)$ with covectors $\xi$ and $\eta$ in relation (13) such that $\xi+\eta=0$ and multiplication on distributions in this space-time manifold is impossible. If we consider the restriction of distributions on the imaginary time $\tau$, then the space-time manifold is Euclidean. As in the above-mentioned case, we always can find densities with covectors $\xi$ and $\eta$ in relation (13) such that $\xi+\eta=0$ (Figure 1(a)). Consequently, multiplication on distributions $w_{(A) \mu}^{a}(x)$ is impossible, too. Thus, instanton density distributions are not contained in the algebra $\mathcal{A}$.

\section{Vacuum tunneling in the Minkowski space.}

Vacuum tunneling of non-abelian gauge theory directly in the Minkowski space has been studied in [54]. In order to connect vacua of different topological indices and to obtain the potential-energy barrier in winding-number space, through which tunneling occurs, the authors introduce a family of intermediate field configurations as

$$
\begin{gathered}
A_{0}=0, \\
\vec{A}(x, t)=\vec{f}(x, \lambda(t)),
\end{gathered}
$$

where $\lambda$ is a parameter describing the field configuration within the family. The initial potential is $\vec{A}^{(1)}=\left.\vec{f}\right|_{\lambda=\lambda_{1}}$ and the terminal potential is $\vec{A}^{(2)}=\left.\vec{f}\right|_{\lambda=\lambda_{2}}$. The function $\vec{f}$ varies continuously from $\vec{A}^{(1)}$ to $\vec{A}^{(2)}$ as $\lambda$ varies from $\lambda_{1}$ to $\lambda_{2}$. The WKB vacuum-tunneling amplitude has been found from differential equations in which the parameter $\lambda(t)$ is the dynamical variable.

But, the attempt to generalize the above-mentioned family of intermediate field configurations on density distributions $w_{(A) \mu}^{a}(x)$ of quantum fields results in impossibility to define multiplication on these densities. Indeed, the motion along the curve $\lambda(t)$ (24) in the winding-number space is possible in forward and backward directions. Consequently, the time reversal $T$ inverts the covector $\xi \in \mathrm{WF}\left(w_{(A) \mu}^{a}\right) \subset T^{*} \mathcal{M}$ and one can always find densities $w_{(A) \mu}^{a(1)}(x)$ and $w_{(A) \mu}^{a(2)}(x)$ with covectors $\xi$ and $\eta$, relatively, in relation (13) such that $\xi+\eta=0$. Multiplication is only partial defined and, therefore, one can conclude that instanton density distributions must be excluded from the algebra $\mathcal{A}$. As it is shown in Section $\mathrm{V}$, these distributions cannot define ideals and points of the space-time manifold $\mathcal{M}$. 
The additional term $\theta Q$ in the action $S(23)$ breaks $P$ and $C P$ invariance and contributes directly to the neutron electric dipole moment [51] [56]. According to the above-mentioned consideration, instanton distributions are impossible and, therefore, tunneling effects between different topological vacua $|n\rangle$ do not occur. This leads to a degeneration of the energy density of the $\theta$-vacuum (22) with respect to the phase $\theta$. The vacuum energy density becomes $\theta$-invariant. In order to achieve the $\theta$-invariance in the action $S$ (23), one should require that the Pontryagin index $Q$ is equal to zero. This leads to the zero value of the neutron electric dipole moment.

Thus, in Sections III and IV, we define the multiplication on distributions of the quantum-field densities and construct the commutative density distribution algebra $\mathcal{A}$. Multiplication is well-defined operation for all elements of the algebra. Fermion, boson (Higgs) and gauge field density distributions with singularities in the future light cone $\Gamma^{(f)}$ satisfying the restriction requirements are generators of the algebra $\mathcal{A}$. Spectrum of the density distribution algebra $\mathcal{A}$ and the scheme $\left(\mathcal{M}, \mathcal{A}_{\mathcal{M}}\right)$ are considered in the next section.

\section{Ideals and Spectrum of the Density Distribution Algebra. Scheme $\left(\mathcal{M}, \mathcal{A}_{\mathcal{M}}\right)$}

For definition of the scheme $\left(\mathcal{M}, \mathcal{A}_{\mathcal{M}}\right)$ contained the spectrum of the commutative density distribution algebra $\mathcal{A}$ isomorphic to the space-time manifold $\mathcal{M}$, it needs to carry out localization and to determine the sheaf of structure algebras and the spectrum of the algebra $\mathcal{A}$. To this end, we define prime and maximal ideals of this algebra. The algebra $\mathcal{A}$ consists of density distributions $w^{\zeta}(x)$ with singularities in the closed future light cone subset, $\mathrm{WF}\left(w^{\zeta}\right) \subset \Gamma^{(f)} \subset T^{*} \mathcal{M}$. The complement of the future light cone subset $\Gamma^{(f)}$ is the open cone subset $\bar{\Gamma}^{(f)}$. In the cone subset $\bar{\Gamma}^{(f)}$ the densities $w^{\zeta}(x)$ are $C^{\infty}$-smooth functions. We define the maximal ideal at the point $x_{0}$ as the set of distributions equal to zero at the point $x_{0}$ in the cone subset $\bar{\Gamma}^{(f)}$ (Figure 2)

$$
m_{x_{0}}=\left\{w^{\zeta}(x) \mid \lim _{x \rightarrow x_{0}} w^{\zeta}(x)=0 \text { in } \bar{\Gamma}^{(f)}\right\} .
$$

$p$ is called a prime ideal if for all distributions $w_{1}(x)$ and $w_{2}(x) \in A$ with $w_{1}(x) w_{2}(x) \in p$ we have $w_{1}(x) \in p$ or $w_{2}(x) \in p$ [57] [58]. Every maximal ideal $m_{x}$ is prime.

In the process of localization of the algebra $\mathcal{A}$ we find a local algebra contained only information about the behavior of density distributions $w^{\zeta}(x)$ near the point $x$ of the space-time manifold $\mathcal{M}$. We consider the case of maximal ideals. Then, the local algebra $\mathcal{A}_{x}$ is defined as the commutative algebra consisting of fractions of density distributions $w_{1}(x)$ and $w_{2}(x)$

$$
\mathcal{A}_{x}=\left\{\frac{w_{1}(x)}{w_{2}(x)} \mid w_{1}(x), w_{2}(x) \in \mathcal{A}, w_{2}(x) \notin m_{x}\right\},
$$




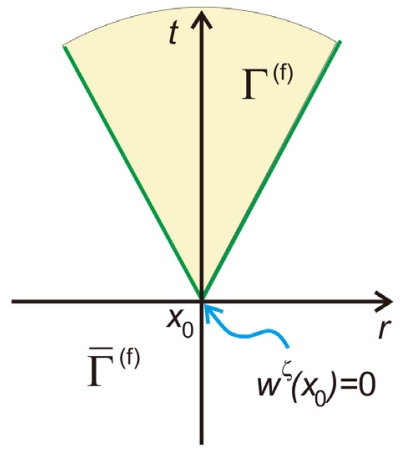

Figure 2. Maximal ideal is defined as the set of distributions equal to zero at the point $x_{0}$ in the cone subset $\bar{\Gamma}^{(f)}$, which is complement of the future light cone.

where $m_{x}$ is the maximal ideal at the point $x$. The fraction $w_{1}(x) / w_{2}(x)$ is the equivalence class defined as

$$
\frac{w_{1}(x)}{w_{2}(x)}=\frac{w_{1}^{\prime}(x)}{w_{2}^{\prime}(x)},
$$

if there exists the distribution $v(x) \in \mathcal{A}, v(x) \notin m_{x}$ such that

$$
v(x)\left(w_{1}(x) w_{2}^{\prime}(x)-w_{2}(x) w_{1}^{\prime}(x)\right)=0 .
$$

Operations on the local algebra $\mathcal{A}_{x}(25)$ look identical to those of elementary algebra

$$
\frac{w_{1}(x)}{w_{2}(x)}+\frac{w_{1}^{\prime}(x)}{w_{2}^{\prime}(x)}=\frac{w_{1}(x) w_{2}^{\prime}(x)+w_{1}^{\prime}(x) w_{2}(x)}{w_{2}(x) w_{2}^{\prime}(x)}
$$

and

$$
\frac{w_{1}(x)}{w_{2}(x)} \cdot \frac{w_{1}^{\prime}(x)}{w_{2}^{\prime}(x)}=\frac{w_{1}(x) w_{1}^{\prime}(x)}{w_{2}(x) w_{2}^{\prime}(x)} .
$$

Algebras $\mathcal{A}_{U_{i}}$ on open sets $U_{i} \subset M$

$$
\mathcal{A}_{U_{i}}=\bigcap_{x \in U_{i}} \mathcal{A}_{x}
$$

determine the structure sheaf [59] $\mathcal{A}_{M}=\left\{\mathcal{A}_{U_{i}}\right\}$ on the space-time manifold $\mathcal{M}$. The inverse limit of the structure sheaf $\mathcal{A}_{M}$ coincides with the algebra $\mathcal{A}$

$$
\mathcal{A}=\lim _{U_{i} \in M} \mathcal{A}_{U_{i}},
$$

where $\left\{U_{i}\right\}$ is the open covering of $\mathcal{M}$.

The spectrum of the algebra $\mathcal{A}$, denoted by $\operatorname{Spec}(A)$, is the set of all prime ideals of $\mathcal{A}$, equipped with the Zariski topology [22] [59] [60]. The prime ideals correspond to irreducible subvarieties of the space $\operatorname{Spec}(A)$. Maximal ideals of the algebra $\mathcal{A}$ correspond to points.

The structure sheaf and the spectrum of the algebra $\mathcal{A}$ are used in definition of schemes [22] [59] [60]. In our case, the scheme over the algebra $\mathcal{A}$ is the pair $\left(\mathcal{M}, \mathcal{A}_{\mathcal{M}}\right)$ such that there exists an open covering $\left\{U_{i}\right\}$ of $\mathcal{M}$ for 
which each pair $\left(U_{i}, \mathcal{A}_{U_{i}}\right)$ is isomorphic to $\left(V_{i}, \mathcal{O}_{V_{i}}\right)$, where $\left\{V_{i}\right\}$ is the open covering of $\operatorname{Spec}(A)$ and $\mathcal{O}_{V_{i}}$ is the restriction of the structure sheaf $\mathcal{O}_{\operatorname{Spec}(A)}$ to each $V_{i}$. As a result, one can say that the local isomorphism $\mathcal{M} \cong \operatorname{Spec}(\mathcal{A})$ imposed by the theory of schemes and by restrictions on multiplication on the quantum-field-density distributions in the algebra $\mathcal{A}$ lead to the dependence of the space-time properties on the matter. The arrow of time, the chirality violation of spinor fields, and the charge conjugation symmetry violation in the boson sector are consequences of this dependence.

\section{Densities of Composite Fields in the Framework of the Diagram Expansion}

Let us consider approximations of Schwinger equation solutions expressed by the diagram expansion and find singularities and wavefronts of composite field densities $w^{\zeta_{(n)} \text { (comp) }}$ in relation (11). For this purpose, it needs to define the generating functional of connected Green's functions $W$. In the abridged notation, the action in the exponent of the formal solution $Z(q)$ (6) of the Schwinger equation can be written in the form

$$
\begin{aligned}
& \frac{i}{\hbar}(S(\Psi)+(q, \Psi))=\sum_{p=1}^{4} \frac{1}{p !} \lambda_{p} \Psi^{p} \\
& \equiv \sum_{p=1}^{4} \frac{1}{p !} \sum_{\zeta_{1} \cdots \zeta_{p}} \int \cdots \int \lambda_{p}^{\zeta_{1} \cdots \zeta_{p}}\left(x_{1} \cdots x_{p}\right) \Psi^{\zeta_{1}}\left(x_{1}\right) \cdots \Psi^{\zeta_{p}}\left(x_{p}\right) \mathrm{d} x_{1} \cdots \mathrm{d} x_{p},
\end{aligned}
$$

where $\lambda_{1}=\bar{q}=\left\{\bar{q}^{\zeta}(x)\right\}=\left\{i q^{\zeta}(x) / \hbar\right\}$ are the auxiliary fields, $\lambda_{2}$ are the operator variables $-\gamma^{\mu} \vec{\partial}_{\mu}-i m^{(\alpha)} c / \hbar,-i \hbar \square-i m^{(n) 2} c^{2} / \hbar$, and $-i \square / \hbar$ acted on fermions, bosons and gauge field potentials, respectively. These operators are defined by Equations (7). $\lambda_{3}$ are constants of the three-particle interactions defined by the Lagrangian terms (3) $\bar{\psi}^{\alpha} \gamma^{\mu} e_{j(a)} T_{a \beta}^{\alpha} A_{\mu}^{a} \psi^{\beta}, \mu_{\alpha \beta n} \bar{\psi}^{\alpha} \varphi^{n} \psi^{\alpha}$, $\mu_{\alpha \beta n}^{*} \bar{\psi}^{\alpha} \varphi^{+n} \psi^{\alpha}$, by terms with $A_{\mu}^{a} A_{\nu}^{b} A_{\rho}^{c}$, and by terms with $A_{\mu}^{a} \varphi^{+n} \varphi^{n}$. The four-particle constants $\lambda_{4}$ are originated from the Lagrangian terms $v\left(\varphi^{+n} \varphi^{n}\right)^{2}$ and $e_{j(a)}^{2} g^{\mu \rho} g^{v \sigma} C_{b c}^{a} C_{d e}^{a} A_{\mu}^{b} A_{r}^{c} A_{\rho}^{d} A_{\sigma}^{e}$.

By definition, the generating functional of connected Green's functions is written as

$$
W\left(\lambda_{1}, \lambda_{2}, \lambda_{3}, \lambda_{4}\right)=\ln Z\left(\lambda_{1}, \lambda_{2}, \lambda_{3}, \lambda_{4}\right)=\ln Z\left(\bar{q}, \lambda_{2}, \lambda_{3}, \lambda_{4}\right),
$$

where $Z$ is defined by relation (1). The Schwinger Equation (4) for the functional $W$ can be written in the form [25]

$$
\lambda_{1}+\sum_{p=2}^{4} \frac{1}{(p-1) !} \lambda_{p} H_{p-1}=0
$$

where

$$
H_{p}=\left(\frac{\delta W}{\delta \lambda_{1}}+\frac{\delta}{\delta \lambda_{1}}\right)^{p} \cdot 1 .
$$

Solution of the Schwinger Equation (28) can be found by iterations in the form of the power series expansion for the functional $W$ with respect to the ver- 
tices $\lambda_{3}, \lambda_{4}$. In the zero approximation $\left(\lambda_{3}=\lambda_{4}=0\right)$ the formal solution (28) of the functional $W$ is written as

$$
W^{(0)}=\frac{\kappa}{2} \operatorname{tr} \ln \left(-\lambda_{2}^{-1}\right),
$$

where $\kappa=1$ for bosons and $\kappa=-1$ for fermions, respectively. $-\lambda_{2}^{-1}\left(x_{1}-x_{2}\right)$ is the kernel of the inverse operator $-\lambda_{2}$ called the propagator. The next iteration can be found by the substitution of $W^{(0)}$ for $W$ in terms $H_{p-1}$ in Equation (28) and so on. The third order vertex approximation of the functional $W\left(\lambda_{1}, \lambda_{2}, \lambda_{3}, \lambda_{4}\right)$ is presented in Figure 3. We assign solid lines to the propagators $-\lambda_{2}^{-1}$. Propagators $-\lambda_{2}^{-1}$ connected to single $\left(\lambda_{1}\right)$, triple $\left(\lambda_{3}\right)$ and quadruple $\left(\lambda_{4}\right)$ vertices must be integrated over space-time variables and must be summed over indices $\zeta$.

Taking into account the causality, which means that an effect cannot occur from a cause that is not in the back (past) light cone of that event [61] [62], we use causal Green's functions and propagators. Thus, propagators $-\lambda_{2}^{-1}=\left\{S_{\beta}^{c \alpha}(x), D_{n l}^{c}(x), D_{0}^{c}(x)\right\}$ can be written in the following form [61].

For fermions

$$
S_{\beta}^{c \alpha}(x)=\left(i \gamma^{\mu} \partial_{\mu}+m^{(\alpha)} c / \hbar\right) \delta_{\beta}^{\alpha} D^{c}(x),
$$

for bosons

$$
D_{n l}^{c}(x)=D^{c}(x) \delta_{n l},
$$

and for gauge field potentials

$$
\begin{array}{lll}
\bullet \cdots=\lambda_{1}=\bar{q} & \ddots \quad=\lambda_{3} \\
-=-\lambda_{2}^{-1} & \ddots & =\lambda_{4}
\end{array}
$$

(a)

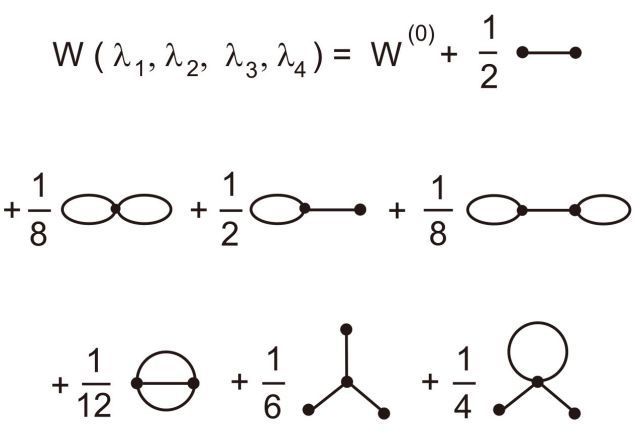

(b)

Figure 3. (a) Propagators $-\lambda_{2}^{-1}$, vertices $\lambda_{1}, \lambda_{3}$ and $\lambda_{4}$. (b) The third order vertex approximation of the functional $W\left(\lambda_{1}, \lambda_{2}, \lambda_{3}, \lambda_{4}\right)$. $W^{(0)}=\frac{\kappa}{2} \operatorname{tr} \ln \left(-\lambda_{2}^{-1}\right)$. 


$$
D_{0}^{c}(x)=\left.D^{c}(x)\right|_{m=0},
$$

where

$$
\begin{gathered}
D^{c}(x)=\theta(t) D^{-}(x)-\theta(-t) D^{+}(x), \\
D^{ \pm}(x)=\frac{1}{4 \pi} \varepsilon(t) \delta(\rho) \mp \frac{m i}{8 \pi \sqrt{\rho}} \theta(\rho)\left[N_{1}(m \sqrt{\rho}) \mp i \varepsilon(t) J_{1}(m \sqrt{\rho})\right] \\
\mp \frac{m i}{4 \pi^{2} \sqrt{-\rho}} \theta(-\rho) K_{1}(m \sqrt{-\rho}), \\
\rho=(c t)^{2}-x^{2} \\
\varepsilon(t)=\theta(t)-\theta(-t) .
\end{gathered}
$$

$N_{1}(x), J_{1}(x), K_{1}(x)$ are Neumann, Bessel and Hankel functions, respectively. Propagators $-\lambda_{2}^{-1}$ (29) are equal to zero in the light-cone exterior and have singularities on the light cone. Taking this into account, we find singularities and wavefronts of composite field densities $w^{\zeta_{(n)} \text { (comp) }}$, which can be written as a derivative of the functional $W$

$$
\begin{aligned}
w^{\zeta(n) \text { (comp) }}(x)= & \left(\frac{i}{\hbar}\right)^{n} \bar{w}^{\zeta_{1}, \cdots, \zeta_{n} \text { (comp) }}(x) \\
= & \left.\left(\frac{i}{\hbar}\right)^{n} \underbrace{\frac{\delta^{n} W}{\delta \bar{q}^{\zeta_{1}}\left(x_{1}\right) \cdots \delta \bar{q}^{\zeta_{n}}\left(x_{n}\right)}}_{n}\right|_{x_{1}, \cdots, x_{n} \rightarrow x} \\
= & \left(\frac{i}{\hbar}\right)^{n} \lim _{x_{1}, \cdots, x_{n} \rightarrow x} \sum_{p} \int \cdots \int\left(-\lambda_{2}^{\zeta_{1}}\right)^{-1}\left(x_{1}-z_{1}\right) \\
& \cdots\left(-\lambda_{2}^{\zeta_{n}}\right)^{-1}\left(x_{n}-z_{n}\right) A^{(p)}\left(z_{1}, \cdots, z_{n}\right) \mathrm{d} z_{1} \cdots \mathrm{d} z_{n},
\end{aligned}
$$

where $n$ is the number of fields, $A^{(p)}\left(z_{1}, \cdots, z_{n}\right)$ is the residual part of the diagram which is depicted by the index $p$. Summation is drawn out over all diagrams. Differentiation of the functional $W$ with respect to auxiliary fields $\bar{q}^{\zeta}$ results in the removal of the corresponding $\lambda_{1}$-vertices in diagrams. In Figure 4 hollow single vertices denote the removed $\lambda_{1}$-vertices and, therefore, the corresponding differentiation operation. For these vertices the integration over space-time variables and the summation over indices $\zeta$ must be dropped out. In this case, singularities of derivations of the functional $W(30)$ are determined by propagators $-\lambda_{2}^{-1}$ (29) connected with hollow single vertices and the composite field densities $w^{\zeta_{(n)} \text { (comp) }}$ have singularities on the light cone. Thus, wavefronts $\operatorname{WF}\left(w^{\zeta_{(n)}(\text { comp })}\right)$ in relation (11) are included in the characteristics $\operatorname{Char}\left(\mathcal{N}_{\zeta}^{\eta}\right)$ and wavefront sets of quantum-field density distributions are located on the light cone.

Now we return to relation (11) and consider a possible extension of the wavefront set $\mathrm{WF}\left(w^{\zeta}\right)$ formed by wavefronts $\mathrm{WF}\left(w^{W_{(n)}(\mathrm{comp})}\right)$ of composite fields. In other words, can wavefronts $\mathrm{WF}\left(w^{\zeta_{(n)}(\mathrm{comp})}\right)$ of composite fields vary the wavefront set $\operatorname{WF}\left(w^{\zeta}\right)$ without loss of multiplication in the algebra $\mathcal{A}$ ? 


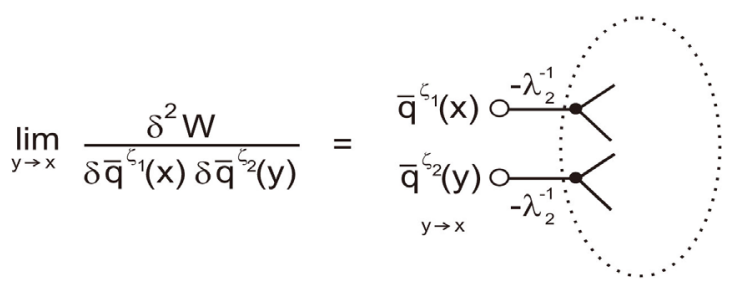

Figure 4. Differentiation of the functional $W$ with respect to the auxiliary fields $\bar{q}^{\zeta_{1}}(x)$ and $\bar{q}^{\zeta_{2}}(y)$. Hollow single vertices denote the differentiation operation. The dotted line bounds residual part of the diagram.

According to the above-mentioned, one can see that the only possible case, in which the multiplication of distributions of quantum-field densities can be defined, is the case, when the quantum fields are in the space-time manifold $\mathcal{M}$ with the structure group $S O(3,1)$. Let us assume that wavefronts of $w^{\zeta_{(n)}(\text { comp) }}(x)$ are in the space-like region $\Gamma^{(s)}$ (Figure $1(\mathrm{~b})$ ) and wavefronts $\mathrm{WF}\left(w^{\zeta(n)(\text { comp })}\right)$ take part to the wavefront $\mathrm{WF}\left(w^{\zeta}\right)$. The transformation $f$ of the space-time manifold $\mathcal{M}$ induced by a rectilinear motion transforms the wavefront of densities $\mathrm{WF}\left(w^{\zeta}\right)$ in relation (14) such that in the space-like region $\Gamma^{(s)}$ the covector inversion $\xi \mapsto-\xi \in \mathrm{WF}\left(w^{\zeta}\right)$ can be reached by the arcwise connected part of the group $S O(3,1)$. Consequently, the extension of the wavefront set $\operatorname{WF}\left(w^{\zeta}\right)$ on the space-like region $\Gamma^{(s)}$ should be ignored because the multiplication on these distributions is impossible. The extension on the past light cone $\Gamma^{(p)}$ should be ignored too because one can find covectors $\xi_{1} \in \Gamma^{(f)}$ and $\xi_{2} \in \Gamma^{(p)}$ such that $\xi_{1}+\xi_{2}=0$. Thus, the multiplication on the densities $w^{\zeta}(x)$ can be defined in the only case, in which the densities are distributions with the wavefront set $\operatorname{WF}\left(w^{\zeta}\right) \in \Gamma^{(f)}$.

\section{Diagram Expansion with Respect to the A-Algebra Variables}

The multiplication on the commutative density distribution algebra $\mathcal{A}$ gives us possibility to define differentiation of functionals of $\mathcal{A}$-algebra variables and to construct diagram expansion with respect to densities. Let us assume that functionals can be given in the form of power series with respect to the densities $w=\left\{w^{\zeta_{1}}(x), \cdots, w^{\zeta_{m}}(x)\right\} \in \mathcal{A}$

$$
R(w)=\sum_{n=0}^{\infty} \sum_{\zeta_{1} \cdots \zeta_{n}} \int \cdots \int F^{\zeta_{1} \cdots \zeta_{n}}\left(x_{1} \cdots x_{n}\right) w^{\zeta_{1}}\left(x_{1}\right) \cdots w^{\zeta_{n}}\left(x_{n}\right) \mathrm{d} x_{1} \cdots \mathrm{d} x_{n} .
$$

The differentiation of the functional $R(w)$ with respect to the density $w^{\xi_{p}}\left(x_{p}\right)$ is reduced to the elimination of the density $w^{\xi_{p}}\left(x_{p}\right)$ and to the dropping out the integral over variables $x_{p}$ in the power series (31)

$$
\begin{aligned}
& \frac{\delta R(w)}{\delta w^{\xi_{p}}\left(x_{p}\right)}=\sum_{n=0}^{\infty} \sum_{\zeta_{1}, \cdots, \xi_{p}, \cdots, \zeta_{n}} \int \cdots \int F^{\zeta_{1} \cdots \zeta_{n}}\left(x_{1} \cdots x_{n}\right) \\
& \times \prod_{k=0}^{p-1} \kappa_{p k} \underbrace{w^{\zeta_{1}}\left(x_{1}\right) \cdots \hat{w}^{\xi_{p}}\left(x_{p}\right) \cdots w^{\zeta_{n}}\left(x_{n}\right)}_{n-1} \mathrm{~d} x_{1} \cdots \mathrm{d} \hat{x}_{p} \cdots \mathrm{d} x_{n},
\end{aligned}
$$


where the mark ${ }^{\wedge}$ points out that the given variable must be dropped. The summation over indices $\zeta_{1}, \cdots, \hat{\xi}_{p}, \cdots, \zeta_{n}$ in relation (32) is performed over all sets $\left\{\hat{\xi}_{p}, \zeta_{2}, \cdots, \zeta_{n}\right\}, \cdots,\left\{\zeta_{1}, \cdots, \zeta_{n-1}, \hat{\xi}_{p}\right\}$. Since the densities $w^{\zeta_{i}}\left(x_{i}\right)$ can be Grassmanian variables, we define the differentiation as left one. The term $\prod_{k=0}^{p-1} \kappa_{p k}$ appears from permutations between densities $w^{\zeta_{i}}\left(x_{i}\right)$ during the differentiation. $\quad \kappa_{p k}=(-1)^{\operatorname{deg} w^{p} \cdot \operatorname{deg} w^{k}}$ depends on parity degrees $\operatorname{deg} w^{p}$, $\operatorname{deg} w^{k}$ of densities $w^{\xi_{p}}\left(x_{p}\right), w^{\xi_{k}}\left(x_{k}\right)$.

In order to construct diagram expansion of Schwinger equation solutions with respect to densities $w^{\zeta}(x)$ belonging to the $\mathcal{A}$-algebra, we use the first Legendre transform of the generating functional $W$. The technique of Legendre transforms makes it possible to find anomalous solutions of the Schwinger equations (4) and (28). Anomalous solutions contain nonperturbative information about quantum field models at spontaneous symmetry breaking and at phase transitions [25] [63] [64] [65]. Furthermore, the use of the diagram expansion of the Legendre transform greatly reduces the number of diagrams. The first Legendre transform $Y$ of the functional $W$ is defined as [25]

$$
Y\left(\bar{w} ; \lambda_{2}, \lambda_{3}, \lambda_{4}\right)=W\left(\bar{q}, \lambda_{2}, \lambda_{3}, \lambda_{4}\right)-\sum_{\zeta} \int \bar{w}^{\zeta}(x) \bar{q}^{\zeta}(x) \mathrm{d} x,
$$

where the functional $W$ is defined by relation (27) and

$$
\bar{w}^{\zeta}(x)=\frac{\hbar}{i} w^{\zeta}(x)=\frac{\vec{\delta} W(\bar{q})}{\delta \bar{q}^{\zeta}(x)} .
$$

Taking into account relations (33) and (34), one can obtain the variable $\bar{q}^{\zeta}(x)$ as the functional of the density $\bar{w}^{\zeta}(x)$

$$
\frac{\vec{\delta} Y(\bar{w})}{\delta \bar{w}^{\zeta}(x)}=-\bar{q}^{\zeta}(x) .
$$

The Schwinger Equation (4) for the functional $Y$ can be written in the form [25]

$$
\frac{\vec{\delta} Y}{\delta \bar{w}}=\lambda_{2} \bar{w}+\sum_{p=3}^{4} \frac{1}{(p-1) !} \lambda_{p} H_{p-1}
$$

where in the abridged notation

$$
H_{p}=\left[\bar{w}-\left(\frac{\vec{\delta}^{2} Y}{\delta \bar{w}^{2}}\right)^{-1} \frac{\vec{\delta}}{\delta \bar{w}}\right]^{p-1} \bar{w}
$$

and

$$
\left(\frac{\vec{\delta}^{2} Y}{\delta \bar{w}^{2}}\right)^{-1}
$$

is the kernel of the inverse operator of

$$
\int \frac{\vec{\delta}^{2} Y}{\delta \bar{w}(x) \delta \bar{w}(y)} f(y) \mathrm{d} y .
$$

By analogy with solutions of Equation (28), solution of the Schwinger equa- 
tion (36) can be found by iterations in the form of the power series expansion for the functional $Y$ with respect to the vertices $\lambda_{3}, \lambda_{4}$. In the zero approximation $\left(\lambda_{3}=\lambda_{4}=0\right)$ the formal solution (36) is the Gaussian functional integral and the functional $Y$ is written as

$$
Y^{(0)}\left(\bar{w} ; \lambda_{2}\right)=\frac{\kappa}{2} \operatorname{tr} \ln \left(-\lambda_{2}^{-1}\right)+\frac{1}{2} \bar{w} \lambda_{2} \bar{w},
$$

where $\kappa=1$ for bosons and $\kappa=-1$ for fermions, respectively. The next iteration can be found by the substitution of $Y^{(0)}$ for $Y$ in the term $H_{2}$ in equation (36) and so on. In diagrams describing these iterations propagators $-\lambda_{2}^{-1}$, vertices $\lambda_{3}$ and $\lambda_{4}$ remain the same as shown in diagrams in Section VI. The $\lambda_{1}$ -vertices should be removed from diagrams and the variables $\bar{w}$ should be included. The first order vertex approximation of the functional $Y\left(\bar{w} ; \lambda_{2}, \lambda_{3}, \lambda_{4}\right)$ is presented in Figure 5. We assign wavy lines to the variables $\bar{w}$. Propagators $-\lambda_{2}^{-1}$ and variables $\bar{w}$ connected to triple $\left(\lambda_{3}\right)$ and quadruple $\left(\lambda_{4}\right)$ vertices must be integrated over space-time variables and must be summed over indices $\zeta$.

The anomalous solution is found as an extremum

$$
\left.\frac{\delta \Phi}{\delta \bar{w}^{\zeta}(x)}\right|_{\bar{w}_{0}^{\zeta}}=0
$$

at the stationarity point $\bar{w}_{0}^{\zeta}(x)$ of the functional

$$
\Phi\left(\bar{w} ; \lambda_{1}, \lambda_{2}, \lambda_{3}, \lambda_{4}\right)=Y\left(\bar{w} ; \lambda_{2}, \lambda_{3}, \lambda_{4}\right)+\sum_{\zeta} \int \bar{w}^{\zeta}(x) \bar{q}^{\zeta}(x) \mathrm{d} x .
$$

In order to find the anomalous solution, the Legendre transform $Y$ should be given in the relevant diagram approximation.

The natural next step should be to go from bare to dressed lines in diagrams, which requires analysis based on the second Legendre transform of the functional $W$. But, it should be noted that in the generating functional $W(27)$ coefficients $\lambda_{2}$ are operator variables. Since, differential operators and density distributions $w^{\zeta}(x)$ form a noncommutative algebra, the differentiation of the functional $W$ with respect to $\lambda_{2}$ may be incorrect. This is required accurate definition of the functional derivative $\delta W / \delta \lambda_{2}$ and, consequently, the second Legendre transform. This problem is planed to consider in the next study. In contrast with this case, in models in statistical physics (for example, for the classical non-ideal gas and for the Ising model) coefficients $\lambda_{2}$ are not operator variables and the second Legendre transform is well defined [25].

\section{Conclusions}

In summary, in this paper in the framework of the scheme theory we describe the dependence between quantum fields and properties of the 4-dimensional space-time manifold $\mathcal{M}$. Contrary to algebras of smooth functions, densities of quantum fields, which can be found from the Schwinger equation, are distributions and, in the common case, do not form an algebra. In order to determine 


$$
\begin{aligned}
& \sim=\bar{w} \quad \because \quad=\lambda_{3}
\end{aligned}
$$

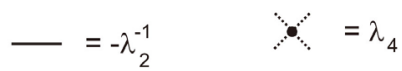

$$
\begin{aligned}
& Y\left(\bar{w} ; \lambda_{2}, \lambda_{3}, \lambda_{4}\right)=Y^{(0)}+\frac{1}{6} \int_{\bar{w}_{2}}^{\bar{w}_{1}} \zeta_{\bar{w}_{3}}^{L_{2}} \\
& +\frac{1}{24} \sqrt[3]{5}+\frac{1}{2} \bigcirc \\
& +\frac{1}{4} \bigcirc x^{5}+\frac{1}{8} \bigcirc
\end{aligned}
$$

(b)

Figure 5. (a) Propagators $-\lambda_{2}^{-1}$, vertices $\lambda_{3}, \lambda_{4}$, and variables $\bar{w}$. (b) The first order vertex approximation of the functional $Y\left(\bar{w} ; \lambda_{2}, \lambda_{3}, \lambda_{4}\right) . Y^{(0)}=\frac{\kappa}{2} \operatorname{tr} \ln \left(-\lambda_{2}^{-1}\right)+\frac{1}{2} \bar{w} \lambda_{2} \bar{w}$.

the commutative algebra $\mathcal{A}$ of distributions of quantum-field densities, ideals and its spectrum, it is necessary to define multiplication on densities and to eliminate those densities, which cannot be multiplied. This leads to essential restrictions imposed on densities forming the algebra $\mathcal{A}$. Taking into account that in the framework of the scheme theory the space-time manifold $\mathcal{M}$ is locally isomorphic to the spectrum of the algebra $\mathcal{A}, \mathcal{M} \cong \operatorname{Spec}(\mathcal{A})$, and points of the manifold $\mathcal{M}$ are defined as maximal ideals of quantum-field density distributions, the restrictions caused by the possibility to define multiplication on the density distributions result in the following properties of the space-time manifold $\mathcal{M}$.

1) The only possible case, when the commutative algebra $\mathcal{A}$ of distributions of quantum-field densities exist, is the case, when the quantum fields are in the space-time manifold $\mathcal{M}$ with the structure group $S O(3,1)$ (Lorentz group). On account of the local isomorphism $\mathcal{M} \cong \operatorname{Spec}(\mathcal{A})$, the quantum fields exist only in the space-time manifold with the one-dimensional time.

2) We must exclude field density distributions with singularities in the past light cone $\Gamma^{(p)}$. The algebra $\mathcal{A}$ consists of the density distributions $w^{\zeta}(x)$ with wavefronts in the closed future light cone subset, $\mathrm{WF}\left(w^{\zeta}(x)\right) \subset \Gamma^{(f)} \subset T^{*} \mathcal{M}$. In this case, we have the one-way direction of time and there is not the symmetry of time on the density distributions. The arrow of time is pointing towards the future.

3) The restrictions caused by multiplication on the density distributions can explain the chirality violation of spinor fields. The densities of right-handed and left-handed fermion states $P \psi_{R}^{\alpha}(x), P \psi_{L}^{\alpha}(x), P \bar{\psi}_{R}^{\alpha}(x), P \bar{\psi}_{L}^{\alpha}(x)$, $C \psi_{R}^{\alpha}(x), C \psi_{L}^{\alpha}(x), C \bar{\psi}_{R}^{\alpha}(x), C \bar{\psi}_{L}^{\alpha}(x)$, where $P$ is the space reflection and $C$ 
is the charge conjugation, are forbidden and are not contained in the algebra $\mathcal{A}$. The commutative algebra $\mathcal{A}$ contains densities $w^{\zeta}(x)$ of states $\psi_{R}^{\alpha}(x)$, $\psi_{L}^{\alpha}(x), \bar{\psi}_{R}^{\alpha}(x), \bar{\psi}_{L}^{\alpha}(x), C P \psi_{R}^{\alpha}(x), C P \psi_{L}^{\alpha}(x), C P \bar{\psi}_{R}^{\alpha}(x), C P \bar{\psi}_{L}^{\alpha}(x)$, their sums and products.

4) For bosons (for example, in the Higgs sector) the densities of states $C \varphi^{n}(x)$ and $C \varphi^{+n}(x)$, where $\varphi^{n}(x) \neq \varphi^{+n}(x)$, must be excluded from the algebra $\mathcal{A}$. The algebra $\mathcal{A}$ contains densities of $\varphi^{n}(x)$ and $\varphi^{+n}(x)$. This leads to the charge conjugation symmetry violation and can explain the observed matter-antimatter imbalance.

5) Multiplication on distributions in the density distribution algebra $\mathcal{A}$ imposes restrictions on theoretical models with non-abelian gauge fields. In the framework of the scheme theory instanton distributions are impossible and, therefore, tunneling effects between different topological vacua $|n\rangle$ do not occur. This leads to a degeneration of the energy density of the $\theta$-vacuum with respect to the phase $\theta$, to zero value of the Pontryagin index $Q$ and to zero value of the neutron electric dipole moment.

The well-defined multiplication on the density distributions $w^{\zeta}(x)$ and the commutability of the algebra $\mathcal{A}$ give the possibility to construct diagram expansion with respect to the $\mathcal{A}$-algebra variables. The technique of Legendre transforms makes it possible to find anomalous solutions of the Schwinger equation.

\section{Conflicts of Interest}

The author declares no conflicts of interest regarding the publication of this paper.

\section{References}

[1] Bars, I. (2000) Physical Review D, 62, Article ID: 046007. https://doi.org/10.1103/PhysRevD.62.105018

[2] Bars, I. (2006) Physical Review D, 74, Article ID: 085019. https://doi.org/10.1103/PhysRevD.74.085019

[3] Bars, I., Terning, J. and Nekoogar, F. (2010) Extra Dimensions in Space and Time. Springer, New York. https://doi.org/10.1007/978-0-387-77638-5

[4] Tegmark, M. (1997) Classical and Quantum Gravity, 14, L69-L75. https://doi.org/10.1088/0264-9381/14/4/002

[5] Chappell, J.M., Hartnett, J.G., Iannella, N., Iqbal, A. and Abbott, D. (2006) Frontiers in Physics, 4, Article 44. https://doi.org/10.3389/fphy.2016.00044

[6] Velev, M.V. (2012) Physics Essays, 25, 403-438. https://doi.org/10.4006/0836-1398-25.3.403

[7] Lebowitz, J.L. (1993) Physics Today, 46, 32-38. https://doi.org/10.1063/1.881363

[8] Kiefer, C. and Zeh, H.D. (1995) Physical Review D, 51, 4145-4153. https://doi.org/10.1103/PhysRevD.51.4145

[9] Zeh, H.D. (2010) The Physical Basis of The Direction of Time (The Frontiers Collection). 5th Edition, Springer, Berlin. 
[10] Jennings, D. and Rudolph, T. (2010) Physical Review E, 81, Article ID: 061130. https://doi.org/10.1103/PhysRevE.81.061130

[11] Mersini-Houghton, L. and Vaas, R. (2012) The Arrows of Time. A Debate in Cosmology. Springer, Berlin. https://doi.org/10.1007/978-3-642-23259-6

[12] Bousso, R. (2012) Physical Review D, 86, Article ID: 123509. https://doi.org/10.1103/PhysRevD.86.123509

[13] Smolin, L. (2013) Time Reborn: From the Crisis in Physics to the Future of the Universe. Houghton Mifflin Harcourt, New York.

[14] Huang, K. (1982) Quarks, Leptons and Gauge Fields. World Scientific, Singapore. https://doi.org/10.1142/0001

[15] Cheng, T.-P. and Li, L.-F. (1984) Gauge Theory of Elementary Particle Physics. Clarendon Press, Oxford.

[16] Barron, L.D. (2012) Chirality, 24, 957-958. https://doi.org/10.1002/chir.22106

[17] Shu, J. and Zhang, Y. (2013) Physical Review Letters, 111, Article ID: 091801. https://doi.org/10.1103/PhysRevLett.111.091801

[18] Cirigliano, V., Dekens, W., de Vries, J. and Mereghetti, E. (2016) Physical Review D, 94, Article ID: 016002. https://doi.org/10.1103/PhysRevD.94.034031

[19] Carena, M., Ellis, J., Lee, J.S., Pilaftsis, A. and Wagner, C.E.M. (2016) Journal of High Energy Physics, 123, 38. https://doi.org/10.1007/JHEP02(2016)123

[20] Englert, Ch., Nordström, K., Sakurai, K. and Spannowsky, M. (2017) Physical Review D, 95, Article ID: 015018. https://doi.org/10.1103/PhysRevD.95.015018

[21] Goodsell, M.D. and Staub, F. (2017) The European Physical Journal C, 77, 46. https://doi.org/10.1140/epjc/s10052-017-5259-x

[22] Grothendieck, A. (1961-1967) Eléments de Géométrie Algébrique, I-IV. Publications Mathématiques de IIHES, 4, 8, 11, 17, 20, 24, 28 and 32.

[23] Cartier, P. (2001) Bulletin of the American Mathematical Society, 38, 389-408. https://doi.org/10.1090/S0273-0979-01-00913-2

[24] Schwinger, J. (1951) Proceedings of the National Academy of Sciences, 37, 452-459. https://doi.org/10.1073/pnas.37.7.452

[25] Vasiliev, A.N. (1998) Functional Methods in Quantum Field Theory and Statistical Physics. Gordon and Breach, London.

[26] Lutsev, L.V. (2007) Journal of Physics A: Mathematical and Theoretical, 40, 11791-11814. https://doi.org/10.1088/1751-8113/40/39/007

[27] Lutsev, L.V. (2009) Diagram Technique for Quantum Models with Internal Lie-Group Dynamics. In: Levy, M.B., Ed., Mathematical Physics Research Developments, Nova Science Publishers, New York, 141-188.

[28] Hörmander, L. (1971) Acta Mathematica, 127, 79-183. https://doi.org/10.1007/BF02392052

[29] Radzikowski, M. (1996) Communications in Mathematical Physics, 179, 529-553. https://doi.org/10.1007/BF02100096

[30] Brunetti, R. and Fredenhagen, K. (2000) Communications in Mathematical Physics, 208, 623-661. https://doi.org/10.1007/s002200050004

[31] Strohmaier, A., Verch, R. and Wollenberg, M. (2002) Journal of Mathematical Physics, 43, 5514-5530. https://doi.org/10.1063/1.1506381

[32] Brouder, Ch., Dang, N.V. and Helein, F. (2014) Journal of Physics A: Mathematical and Theoretical, 47, Article ID: 443001. 
https://doi.org/10.1088/1751-8113/47/44/443001

[33] Treves, F. (1982) Introduction to Pseudo-Differential and Fourier Integral Operators. Plenum Press, New York and London.

[34] Dubrovin, B.A., Fomenko, A.T. and Novikov, S.P. (1992) Modern Geometry-Methods and Applications. Part I. The Geometry of Surfaces, Transformation Groups, and Fields. Second Edition, Springer, New York.

[35] Hörmander, L. (1985) The Analysis of Linear Partial Differential Operators. Volume 1: Distribution Theory and Fourier Analysis. Springer-Verlag, Berlin. https://doi.org/10.1007/978-3-662-30724-3 1

[36] Hörmander, L. (1985) The Analysis of Linear Partial Differential Operators. Volume 4: Fourier Integral Operators. Springer-Verlag, Berlin.

[37] Davydov, A.S. (1976) Quantum Mechanics. Pergamon Press, Oxford.

[38] Englert, F. and Brout, R. (1964) Physical Review Letters, 13, 321-323. https://doi.org/10.1103/PhysRevLett.13.321

[39] Higgs, P.W. (1964) Physics Letters, 12, 132-133. https://doi.org/10.1016/0031-9163(64)91136-9

[40] Higgs, P.W. (1964) Physical Review Letters, 13, 508-509. https://doi.org/10.1103/PhysRevLett.13.508

[41] Gunion, J.F. and Haber, H.E. (2003) Physical Review D, 67, Article ID: 075019. https://doi.org/10.1103/PhysRevD.67.075019

[42] Branco, G.C., Ferreira, P.M., Lavoura, L., Rebelo, M.N., Sher, M. and Silva, J.P. (2012) Physics Reports, 516, 1-102. https://doi.org/10.1016/j.physrep.2012.02.002

[43] Haber, H.E. and Kane, G.L. (1985) Physics Reports, 117, 75-263. https://doi.org/10.1016/0370-1573(85)90051-1

[44] Baer, H. and Tata, X. (2006) Weak Scale Supersymmetry: From Superfields to Scattering Events. Cambridge Univ. Press, Cambridge. https://doi.org/10.1017/CBO9780511617270

[45] Bott, R. (1959) Annals of Mathematics, 70, 313-337. https://doi.org/10.2307/1970106

[46] Dubrovin, B.A., Fomenko, A.T. and Novikov, S.P. (1985) Modern Geometry-Methods and Applications: Part II, the Geometry and Topology of Manifolds. Graduate Texts in Mathematics, Vol. 104, Springer Verlag, New York.

[47] Rajaraman, R. (1982) Solitons and Instantons. An Introduction to Solitons and Instantons in Quantum Field Theory. North-Holland Publishing Company, Amsterdam.

[48] Jackiw, R. and Rebbi, C. (1976) Physical Review Letters, 37, 172-175. https://doi.org/10.1103/PhysRevLett.37.172

[49] Callan, C.G., Dashen, R.F. and Gross, D.J. (1976) Physics Letters, 63B, 334-340. https://doi.org/10.1016/0370-2693(76)90277-X

[50] 't Hooft, G. (1976) Physical Review Letters, 37, 8-11. https://doi.org/10.1103/PhysRevLett.37.8

[51] Wilczek, F. (1978) Physical Review Letters, 40, 279-282. https://doi.org/10.1103/PhysRevLett.40.279

[52] Llewellyn Smith, C.H. (1982) Philosophical Transactions of the Royal Society of London A, 304, 5-22.

[53] Schäfer, T. and Shuryak, E.V. (1998) Reviews of Modern Physics, 70, 323-425. 
https://doi.org/10.1103/RevModPhys.70.323

[54] Bitar, K.M. and Chang, S.-J. (1978) Physical Review D, 17, 486-497. https://doi.org/10.1103/PhysRevD.17.486

[55] de Vega, H.J., Gervais, J.L. and Sakita, B. (1978) Nuclear Physics B, 143, 125-147. https://doi.org/10.1016/0550-3213(78)90451-0

[56] Crewther, R.J., Di Vecchia, P., Veneziano, G. and Witten, E. (1979) Physics Letters, 88B, 123. https://doi.org/10.1016/0370-2693(79)90128-X

[57] Cox, D., Little, J. and O’Shea, D. (1998) Ideals, Varieties, and Algorithms. Springer-Verlag, New York. https://doi.org/10.1007/978-1-4757-2693-0

[58] Lang, S. (2002) Algebra. Graduate Texts in Mathematics. Springer-Verlag, Berlin. https://doi.org/10.1007/978-1-4613-0041-0

[59] Mumford, D. (1999) The Red Book of Varieties and Schemes. Springer-Verlag, Berlin. https://doi.org/10.1007/b62130

[60] Eisenbud, D. and Harris, J. (1998) The Geometry of Schemes. Springer-Verlag, Berlin.

[61] Bogoliubov, N.N. and Shirkov, D.V. (1980) Introduction to the Theory of Quantized Field. John Wiley \& Sons, New York.

[62] Bohm, D. (2005) Causality and Chance in Modern Physics. Taylor and Francis, London.

[63] Vasil'ev, A.N. and Kazanskii (1973) Theoretical and Mathematical Physics, 14, 215-226. https://doi.org/10.1007/BF01029302

[64] De Dominicis, C. and Martin, P.C. (1964) Journal of Mathematical Physics, 5, 14-30. https://doi.org/10.1063/1.1704062

[65] De Dominicis, C. and Martin, P.C. (1964) Journal of Mathematical Physics, 5, 31-59. https://doi.org/10.1063/1.1704064 